\title{
RelA regulates CXCL1/CXCR2-dependent oncogene-induced senescence in murine Kras-driven pancreatic carcinogenesis
}

\author{
Marina Lesina, ${ }^{1}$ Sonja Maria Wörmann, ${ }^{1}$ Jennifer Morton, ${ }^{2}$ Kalliope Nina Diakopoulos, ${ }^{1}$ Olga Korneeva, ${ }^{1}$ Margit Wimmer, ${ }^{1}$ \\ Henrik Einwächter, ${ }^{1}$ Jan Sperveslage, ${ }^{3}$ Ihsan Ekin Demir, ${ }^{4}$ Timo Kehl, ${ }^{5}$ Dieter Saur, ${ }^{1}$ Bence Sipos, ${ }^{6}$ Mathias Heikenwälder, ${ }^{7,8}$ \\ Jörg Manfred Steiner, ${ }^{1,9}$ Timothy Cragin Wang, ${ }^{10}$ Owen J. Sansom, ${ }^{2}$ Roland Michael Schmid, ${ }^{1}$ and Hana Algül' \\ 'II. Medizinische Klinik, Klinikum rechts der Isar, Technische Universität München, Munich, Germany. ² Cancer Research UK Beatson Institute, Department of Pathology, Glasgow, United Kingdom. \\ ${ }^{3}$ Universitätsklinikum Münster, Münster, Germany. ${ }^{4}$ Department of Surgery, Klinikum rechts der Isar, Technische Universität München, Munich, Germany. ${ }^{5}$ German Cancer Research Center (DKFZ),

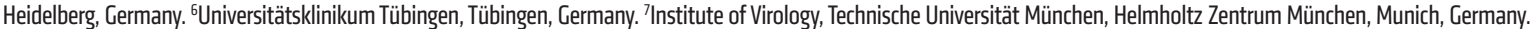 \\ ${ }^{8}$ Division of Chronic Inflammation and Cancer, Cerman Cancer Research Center (DKFZ), Heidelberg, Germany. ${ }^{9}$ Department of Small Animal Clinical Sciences, Texas A\&M University, \\ College Station, Texas, USA. ${ }^{10}$ Division of Digestive and Liver Diseases, Department of Medicine, Irving Cancer Research Center, Columbia University, New York, New York, USA.
}

Tumor suppression that is mediated by oncogene-induced senescence (OIS) is considered to function as a safeguard during development of pancreatic ductal adenocarcinoma (PDAC). However, the mechanisms that regulate OIS in PDAC are poorly understood. Here, we have determined that nuclear RelA reinforces OIS to inhibit carcinogenesis in the Kras mouse model of PDAC. Inactivation of RelA accelerated pancreatic lesion formation in Kras mice by abrogating the senescence-associated secretory phenotype (SASP) gene transcription signature. Using genetic and pharmacological tools, we determined that RelA activation promotes OIS via elevation of the SASP factor CXCL1 (also known as KC), which activates CXCR2, during pancreatic carcinogenesis. In Kras mice, pancreas-specific inactivation of CXCR2 prevented OIS and was correlated with increased tumor proliferation and decreased survival. Moreover, reductions in CXCR2 levels were associated with advanced neoplastic lesions in tissue from human pancreatic specimens. Genetically disabling OIS in Kras mice caused RelA to promote tumor proliferation, suggesting a dual role for RelA signaling in pancreatic carcinogenesis. Taken together, our data suggest a pivotal role for RelA in regulating OIS in preneoplastic lesions and implicate the RelA/CXCL1/CXCR2 axis as an essential mechanism of tumor surveillance in PDAC.

\section{Introduction}

Pancreatic ductal adenocarcinoma (PDAC) is the fourth leading cause of cancer-associated death in Western populations. Morphologically, PDAC is characterized by the presence of pancreatic intraepithelial neoplasia (PanIN) with an activating KRAS mutation occurring in $30 \%$ of precursor lesions and in $100 \%$ of advanced PDAC lesions. Mouse models with pancreas-specific activation of oncogenic Kras recapitulate the features of human PDAC.

As a ubiquitously expressed transcription factor, NF- $\kappa \mathrm{B}$ can regulate the growth and malignancy of many solid tumors, including pancreatic cancer, via the regulation of expression of proinflammatory and prosurvival genes. However, despite extensive characterization of $\mathrm{NF}-\kappa \mathrm{B}$ signaling, the specific functional roles of different members of the NF- $\kappa \mathrm{B}$ family in tumor development are not completely understood. Recently, the role of the kinase IKK2 in PDAC has been explored, demonstrating that IKK exerts its tumor-promoting effects through cross-talk with either Notch1

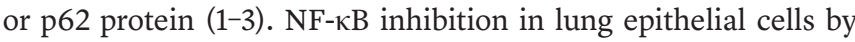

\section{Related Commentary: p. 2799}

Conflict of interest: The authors have declared that no conflict of interest exists. Submitted: January 13, 2016; Accepted: May 13, 2016.

Reference information: / Clin Invest. 2016;126(8):2919-2932. doi:10.1172/JCI86477. genetic deletion of the NF- $\mathrm{BB}$ subunit RelA or IKK2 reduced lung tumorigenesis in a Kras-induced mouse model of lung adenocarcinoma $(4,5)$. In keeping with those reports, overexpression of $\mathrm{I} \kappa \mathrm{B} \alpha$ super-repressor (I $\mathrm{B} \alpha \mathrm{SR}$ ) also resulted in a significantly diminished lung tumor growth, supporting a protumorigenic role of NF- $\kappa \mathrm{B}$ in epithelial cells (6). Although a number of reports display a tumor-promoting role of NF- $\mathrm{KB}$ signaling, it has also been reported to exhibit tumor-suppressing functions in different tumor models. Hepatocyte-specific knockout of IKK $\beta$ resulted in increased tumor development in a chemical-driven model of hepatic carcinogenesis (7). Another study of hepatic carcinogenesis showed that NEMO/IKK $\gamma$-mediated NF- $\kappa \mathrm{B}$ activation in hepatocytes prevented the spontaneous development of hepatic steatosis and hepatocellular carcinoma $(8,9)$, suggesting that components of the NF- $\mathrm{KB}$ pathway play highly pleiotropic roles (10). NF- $\mathrm{kB}$ signaling, when activated, can act as both a tumor promoter and a tumor suppressor. However, the pathogenetic mechanisms by which NF- $\mathrm{BB}$ induces these opposite effects during tumor development remains unclear.

One possibility is the involvement of NF- $\kappa \mathrm{B}$ in the regulation of factors, including CXCL chemokines, IL-6, GM-CSF, and MCP-1, -2 , and -3 , that are subsumed under the term "senescenceassociated secretory phenotype" (SASP) (11). It is now clear that SASP components actively participate in the cellular senescence 
A

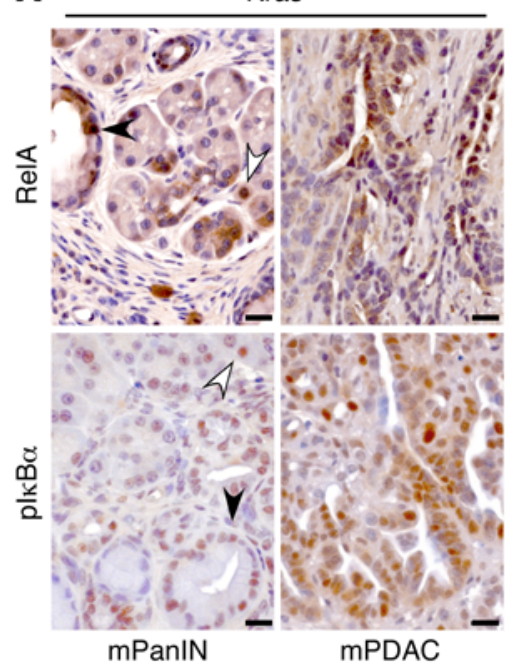

B

C
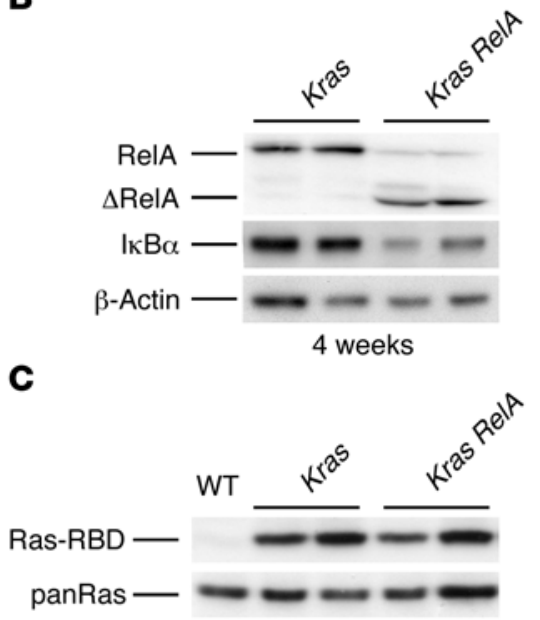

$\mathbf{F}$

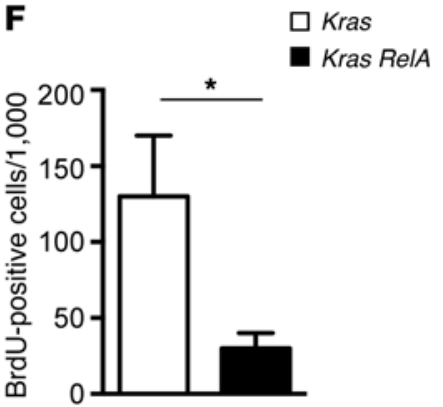

G
D
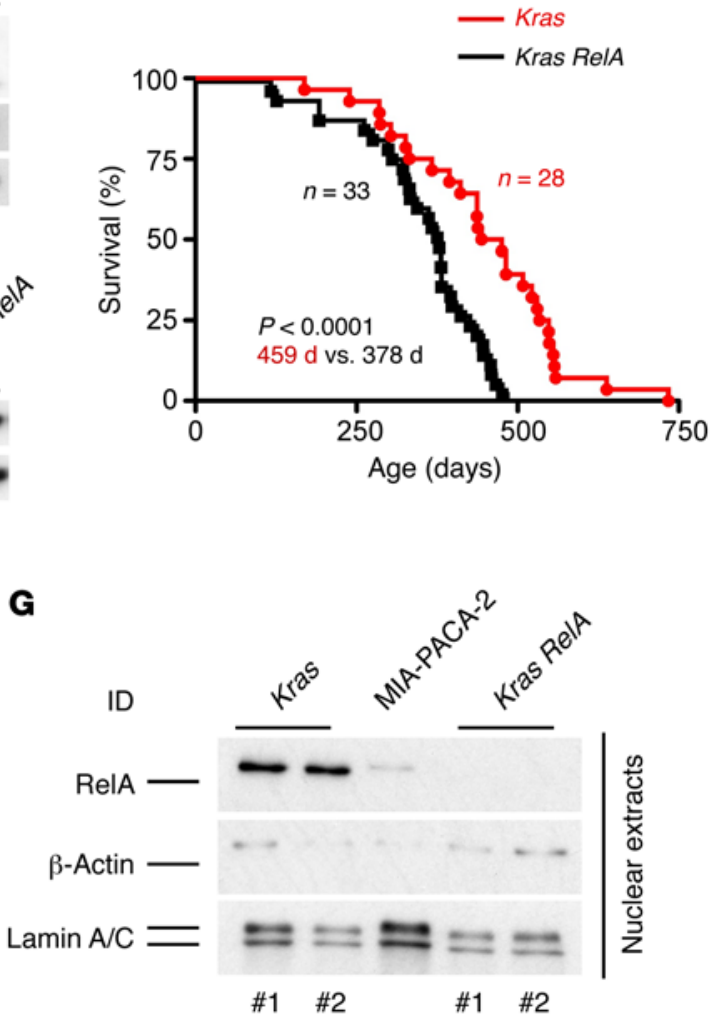

Figure 1. RelA deficiency promotes pancreatic carcinogenesis. (A) IHC analysis of RelA and phosphorylated I $\mathrm{B} \alpha(\mathrm{pl \kappa} B \alpha)$ in pancreata from $K r a s$ mice. Representative images show that RelA and plкB $\alpha$ expression is intense in ductal lesions (black arrowhead), acinar cells (white arrowhead), and PDAC lesions from Kras mice. Scale bars: $20 \mu \mathrm{m}$. (B) Immunoblot analysis of RelA, truncated RelA ( $\triangle \mathrm{RelA}$ ), and IкB $\alpha$ in lysates from 4-week-old Kras and Kras RelA mice. $\beta$-Actin was used as the loading control. (C) Immunoprecipitation of activated Ras (Ras-RBD) in pancreata from mice with the indicated genotypes. Lysate from a WT (LSL-Kras ${ }^{G 120}$ ) mouse served as the negative control. (D) RelA deficiency cooperates with oncogenic Kras ${ }^{6120}$ to influence survival. Kaplan-Meier analysis shows a median survival of 378 days in Kras RelA mice $(n=33$; black line) versus 459 days in Kras mice $(n=28$; red line). $P<0.0001$ by Mantel-Cox log rank test; $n$, number of mice. (E) Tumor incidence was significantly lower in Kras (53.6\%) than in Kras RelA (87.9\%) cohorts. ${ }^{* *} P=0.0041$ by Fisher's exact test. (F) Proliferation index of pancreatic tumors from mice with the indicated genotypes. The number of BrdU-positive cells was counted in 10 fields per mouse. Mean $\pm \mathrm{SD} ; n=3$; ${ }^{*} P<0.05$ by unpaired $t$ test; $n$, number of mice. (G) Immunoblot analysis of nuclear extracts from Kras and Kras RelA primary pancreatic cancer cell lines displays nuclear RelA in tumor cells isolated from Kras but not RelA-deficient Kras mice. MIA-PACA-2 cell line extract served as the positive control. $\beta$-Actin and lamin $A / C$ served as loading controls.

process. Oncogene-induced senescence (OIS) has only recently been shown to govern the carcinogenesis of PDAC (12-16). More specifically, OIS in oncogenic Kras ${ }^{G 12 D}$ mice establishes an incomplete tumor-suppressive program that prevents the expansion of cells that harbor mutant $\operatorname{Kras}(17,18)$. Despite the potential significance of SASP in senescence, little is known about its regulation. Importantly, the impact of SASP on pancreatic carcinogenesis has not been examined directly.

In this study, we aimed to determine the function of RelA in pancreatic cancer in vivo. Surprisingly, our results show that, unlike IKK2 or NEMO, RelA has a tumor-suppressive role. RelA controls the development of pancreatic cancer, because of its function in mediating OIS and immune surveillance. The tumorpromoting functions of RelA are unmasked upon disabling OIS, thus revealing a dual stage function of RelA in the carcinogenesis of PDAC. These findings might prove to be of relevance for future clinical applications.

\section{Results}

Inactivation of RelA increases pancreatic cancer incidence and decreases survival in Kras mice, although tumor proliferation is reduced. Although nuclear RelA is typically detectable in human pancreatic cancer, its functional relevance for this disease is unknown. To determine the function of RelA in pancreatic carcinogenesis, we used the LSL-Kras ${ }^{G 12 D}$ Ptfla-Cre ${ }^{e x 1}$ (hereafter referred to as Kras) mouse model of pancreatic cancer. Nuclear RelA and phosphorylated I $\mathrm{BB} \alpha$ were detectable in Kras mice starting in preneoplastic lesions and adjacent acinar cells and remained visible in established PDAC lesions (Figure 1A). To elucidate the role of canonical NF- $\kappa \mathrm{B}$ signaling in the Kras model, we deleted Rela using a conditional Rela allele (LSL-Kras ${ }^{G 12 D}$ Relaflfl Ptfla-Cre $e^{e x 1}$ mice, hereafter referred to as Kras RelA) (19). Inactivation of RelA in the pancreas had no impact on Ras activity, while less expression of the target gene I $\mathrm{B} \alpha$ was detectable (Figure 1, B and C, and Supplemental Figure 1, A and B; supple- 
A

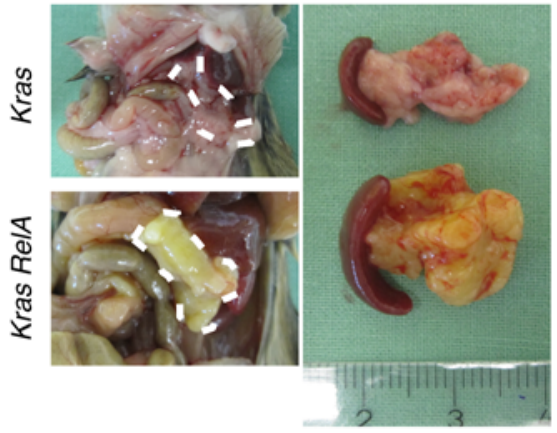

B

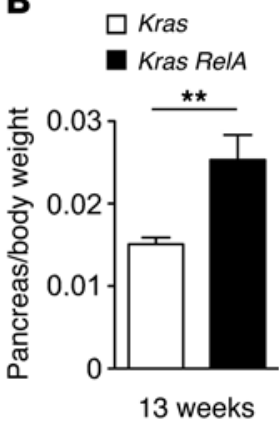

C

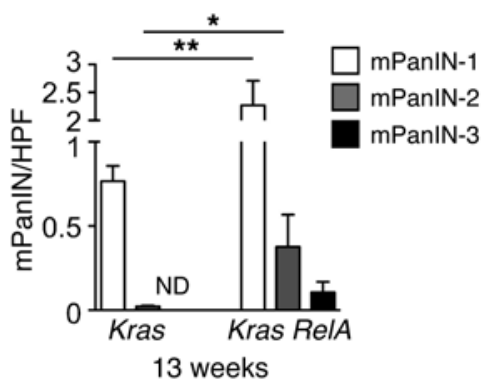

13 weeks

D
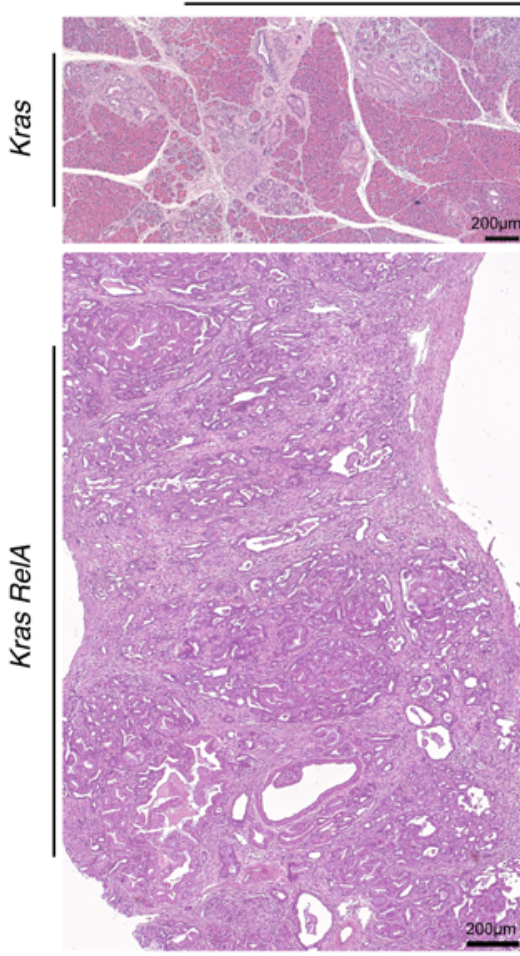

H\&E
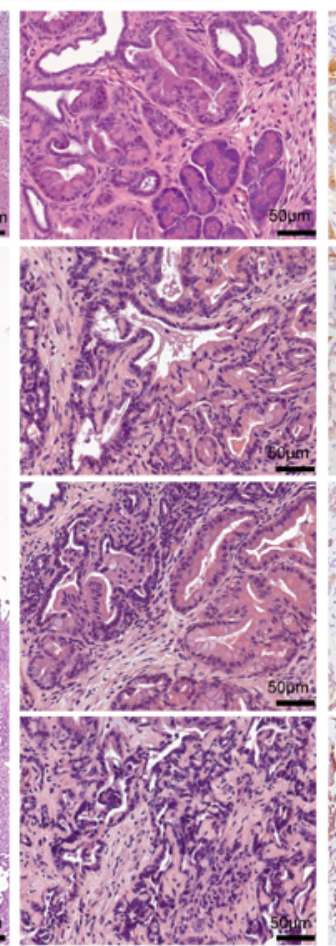

18 weeks
CK19

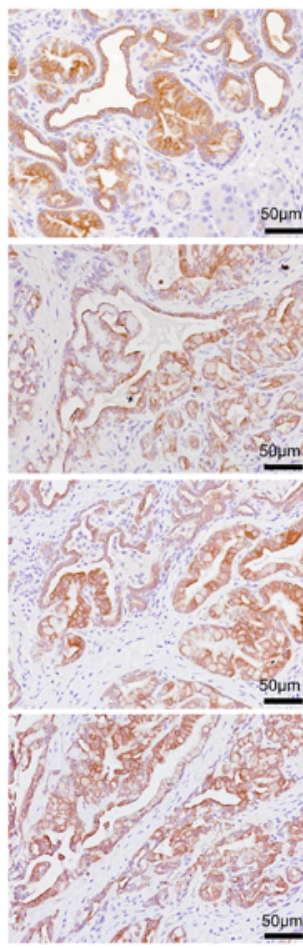

Muc5AC

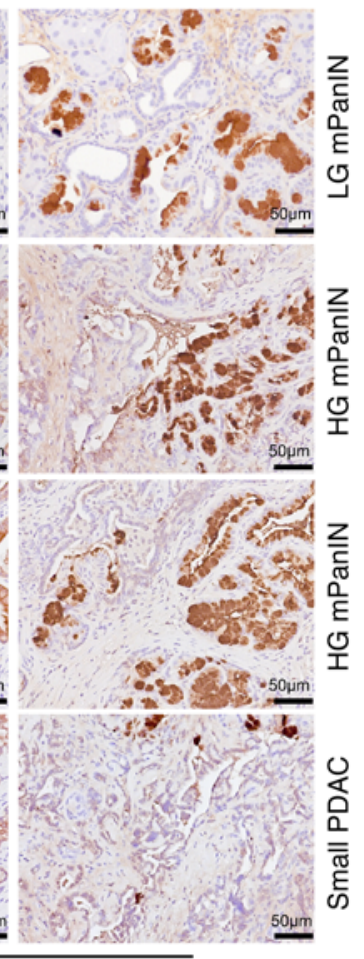

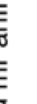

.

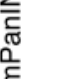


A

Kras

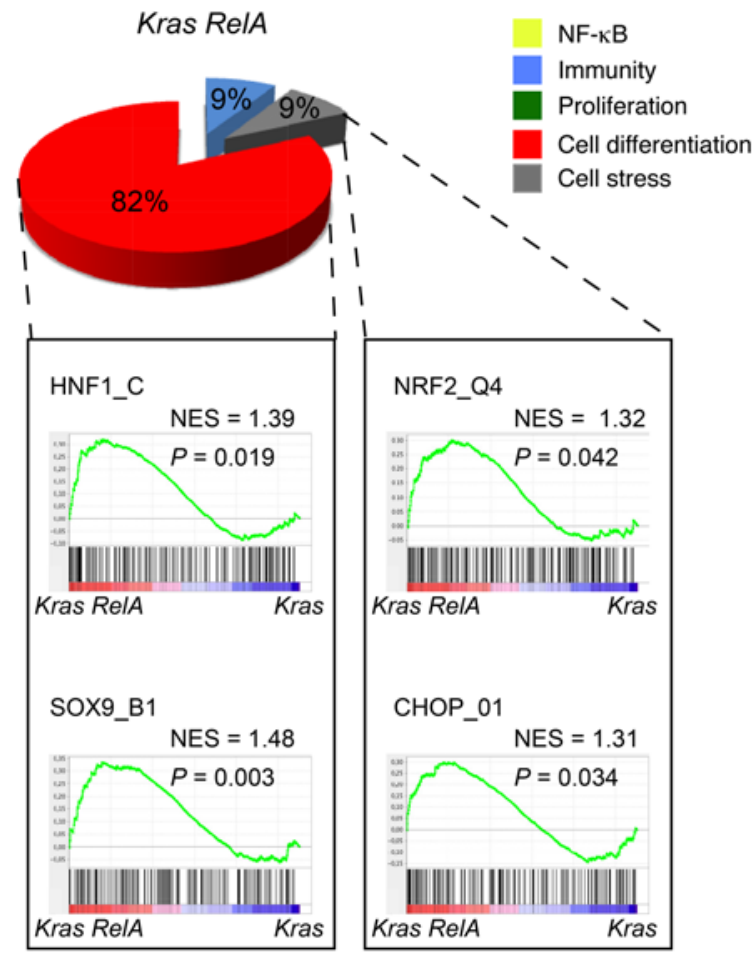

RelA, DNA, Merge

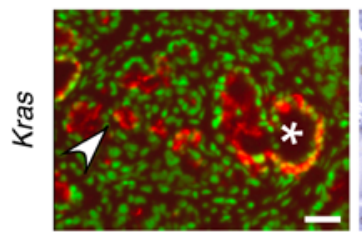

HNF-1 $1 \beta$

SOX9

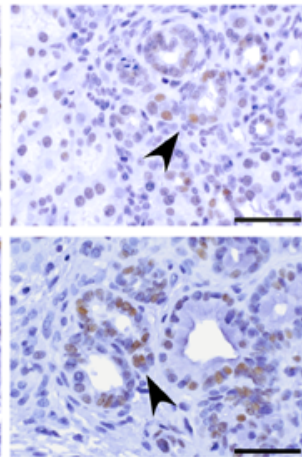

$\gamma-\mathrm{H} 2 \mathrm{AX}$

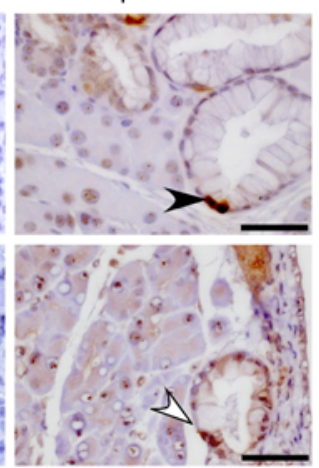

DCFDA

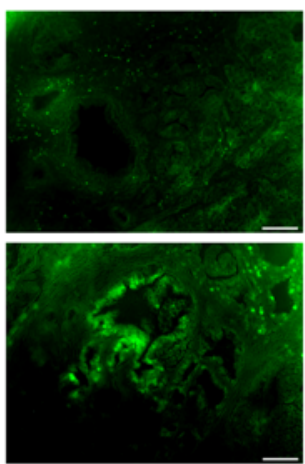

Figure 3. Transcriptomic analysis of Kras RelA pancreata reveals increased cell differentiation and cell stress signaling. (A, top) Classification of upregulated gene sets (NES $\geq 1.2$; nominal $P$ value $<0.05$ ) in pancreata from Kras and Kras RelA mice, based on KEGG and the transcription factor pathway analyses. (A, bottom and boxed) GSEA illustrating enriched gene sets. KEGG analyses illustrating highly enriched gene sets for "chemokine signaling pathways" and "cytokine-cytokine receptor interaction pathways." (B) Immunofluorescent staining of RelA in Kras and Kras RelA mice. White stars indicate ductal lesions, white arrowheads indicate acinar cells. Scale bars: $20 \mu \mathrm{m}$. IHC detection of HNF-1 $\beta$, SOX9, and $\gamma$-H2AX in Kras and Kras RelA mice. Scale bars: $50 \mu \mathrm{m} .2^{\prime}, 7^{\prime}$-Dichlorofluorescein diacetate (DCFDA) staining. Scale bars: $50 \mu \mathrm{m}$. All representative images are shown.

(Supplemental Figure 1H). These results indicate that functional inactivation of RelA accelerates pancreatic cancer development, although proliferation of RelA-deficient tumor cells in established PDAC was significantly reduced.

Taken together, our data suggest an opposite function of RelA compared with IKK2 and NEMO in pancreatic carcinogenesis. RelA seems to play a dual stage-dependent role during tumor development.

Functional inactivation of RelA accelerates progression of murine PanIN (mPanIN) lesions. PDAC is preceded by the evolution of PanIN precursor lesions. Under certain conditions, acinar-to-ductal metaplasia (ADM) might be critical for the development of PanIN lesions. Because functional inactivation of RelA increased tumor incidence while reducing tumor cell proliferation, it seemed very likely that RelA affects mPanIN initiation and progression. Therefore, we evaluated pancreatic carcinogenesis in RelA-deficient Kras mice in more detail. Macroscopically, pancreata of 13-weekold Kras RelA mice appeared enlarged, indurated, and fibrotic when compared with age-matched pancreata of Kras mice (Figure 2, A and B). Histologically, mPanIN progression in Kras RelA mice was significantly accelerated. Indeed, mPanIN-2 and mPanIN-3 lesions were present in Kras RelA mice as early as 13 weeks of age (Figure 2C). IHC examination of Kras RelA pancreata at 18 weeks of age revealed clusters of high-grade $\mathrm{mPanIN}$ lesions alongside small PDACs (Figure 2D). At these time points neither high-grade mPanIN lesions nor focal cancers could be detected in Kras mice (Figure 2D). In addition, pathways that are known to be relevant for ADM formation and mPanIN progression were upregulated in Kras 
A

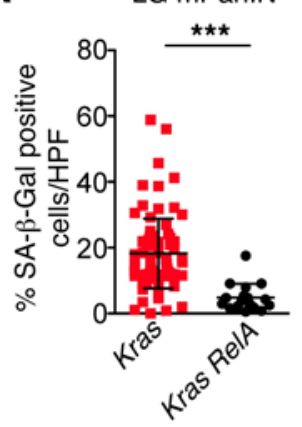

D

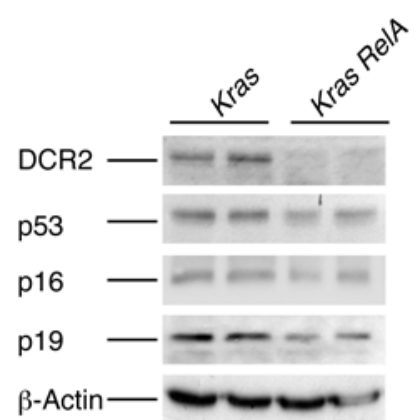

B

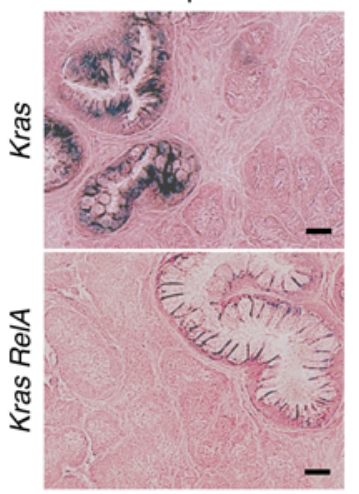

E

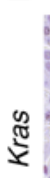

DCR2
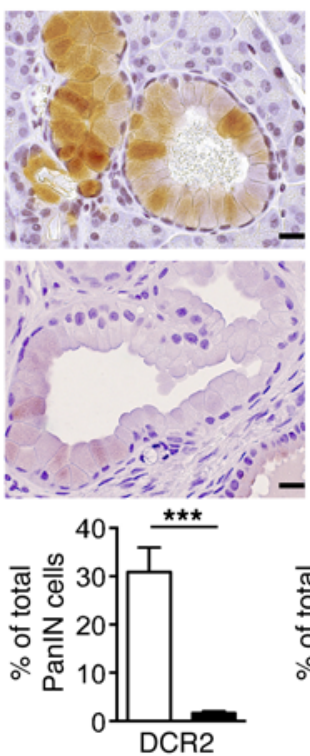

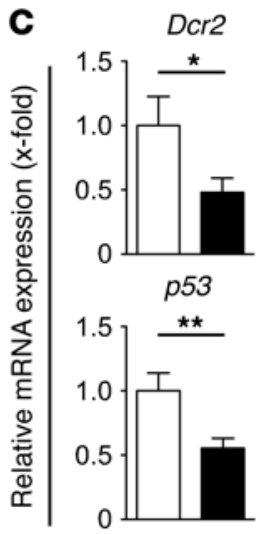

p21

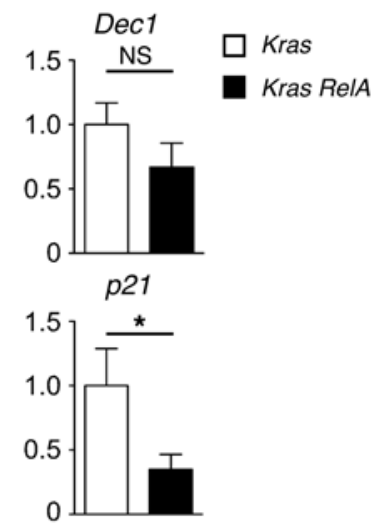

p19
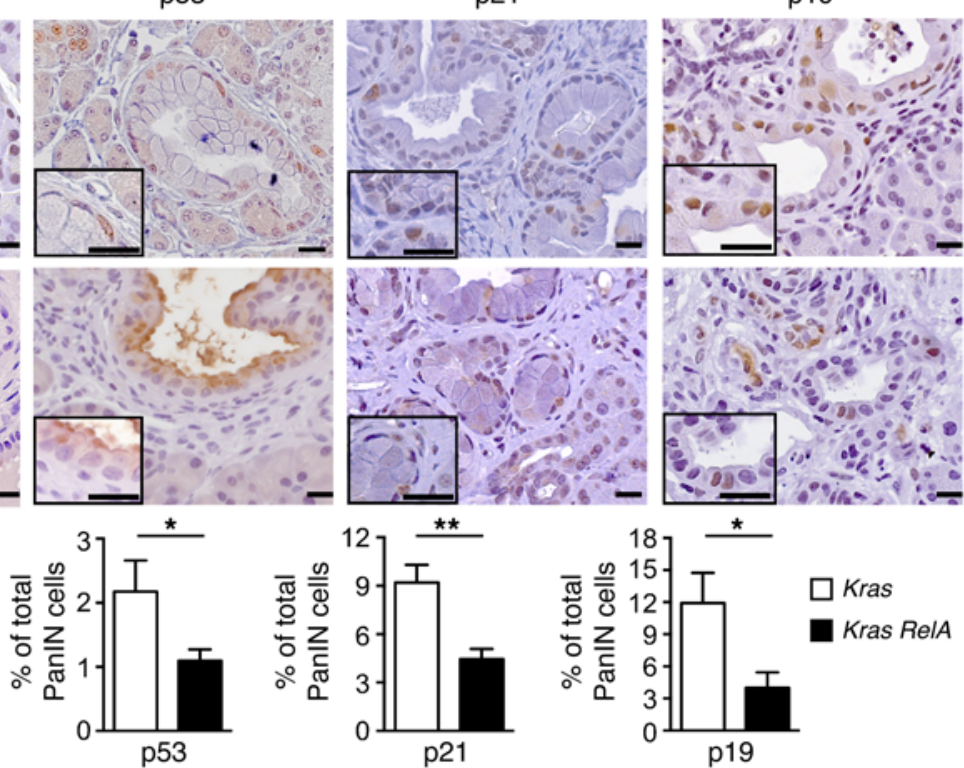

$\square$ Kras

Kras RelA

$\mathbf{F}$

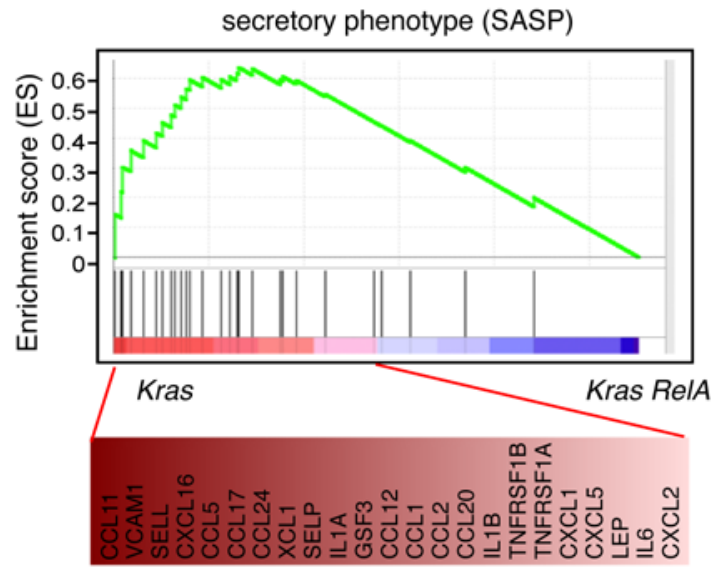

Figure 4. RelA deficiency compromises OIS. (A) Ratio of SA- $\beta$-Gal-positive mPanIN cells to the total number of mPanIN cells in low-grade mPanIN (LG mPanIN) in Kras (6 mice) and Kras RelA (5 mice) was counted per $\times 200$ optical field (high-power field, HPF). Mean \pm SD; $n \geq 50 ;{ }^{* *} P<0.0001$ by Mann-Whitney test; $n$, number of HPFs. (B) Detection of senescence in pancreatic cryosections from Kras and Kras RelA mice using SA- $\beta$-Gal as a marker. Scale bars: $20 \mu \mathrm{m}$. Representative images are shown. (C) Real-time PCR expression analysis of indicated genes in pancreatic lysates from Kras $(n=3)$ and Kras ReIA $(n=3)$ mice. Error bars are shown as SD. Results are the average of 3 independent experiments and are presented as mean $\pm \mathrm{SD} ;{ }^{*} P<0.05,{ }^{*} P<0.005$ by unpaired $t$ test; $n$, number of mice. (D) Pancreatic extracts were collected from Kras and Kras RelA genotypes and analyzed by immunoblot analysis using antibodies against DCR2, p53, p16, or p19. $\beta$-Actin served as loading control. (E) Immunohistological analysis for DCR2, p53, p21, or p19 in Kras $(n=3)$ and Kras RelA $(n=3)$ pancreata. Scale bars: $20 \mu \mathrm{m}$. Insets denote single-positive cells. Representative images are shown. Quantification of DCR2, p53, p21, and p19 positivity in PanIN cells shown at the bottom. Results are presented as mean $\pm \mathrm{SD}$; ${ }^{*} P<0.05$, ${ }^{* *} P<0.005$, ${ }^{* *} P<0.0005$ by unpaired $t$ test; $n$, number of mice. (F) SASP gene set enrichment analysis of pancreatic RNA from Kras $(n=2)$ and Kras RelA $(n=2)$ mice. $n$, number of mice.
RelA mPanIN lesions. These pathways included phosphorylation of ERK1/2, STAT3, and c-Jun (Supplemental Figure 2).

These data reveal that inactivation of RelA in Kras mice accelerates pancreatic carcinogenesis. Classical NF- $\kappa \mathrm{B} /$ RelA signaling seems to inhibit ADM and progression of mPanIN lesions, exerting a specific tumor suppressor function in pancreatic carcinogenesis.

Loss of functional RelA abrogates NF- $\kappa B$ signatures, but increases signatures of cell stress and ADM. To further elucidate the mecha- nisms that promote pancreatic carcinogenesis in Kras RelA mice, we performed microarray analysis with RNA from RelA-proficient and -deficient Kras total pancreata. In order to ensure homogeneous sampling, we used mice younger than 4 weeks of age, as pancreata in both groups did not reveal relevant morphological differences at this time point (Supplemental Figure 3A). For all the transcriptional analysis we compared Kras RelA to Kras pancreas or vice versa. Thus the control for Kras RelA was Kras pancreas with RelA proficiency. 

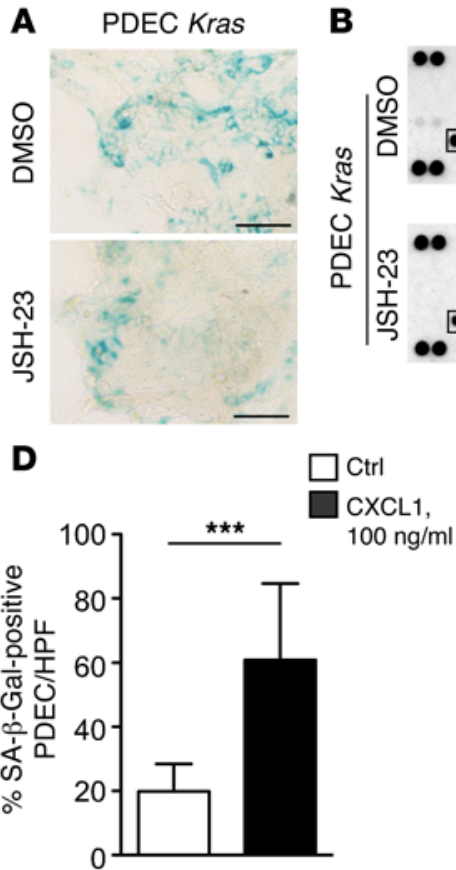

B

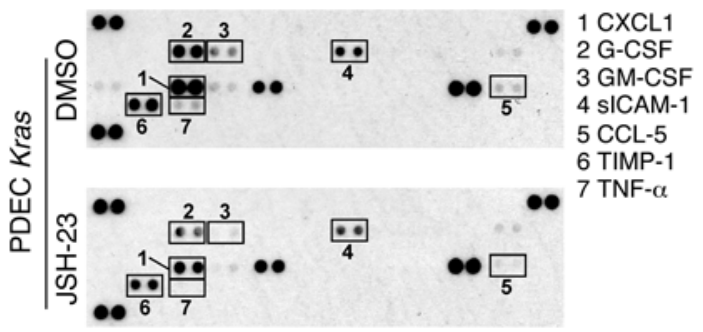

E
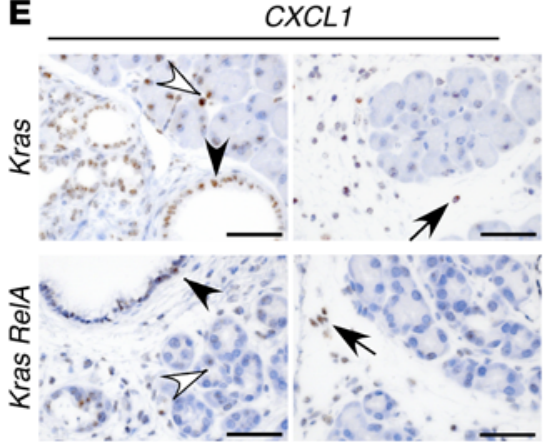

In situ

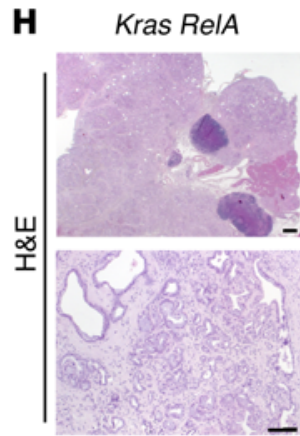

Kras RelA IL-8tg
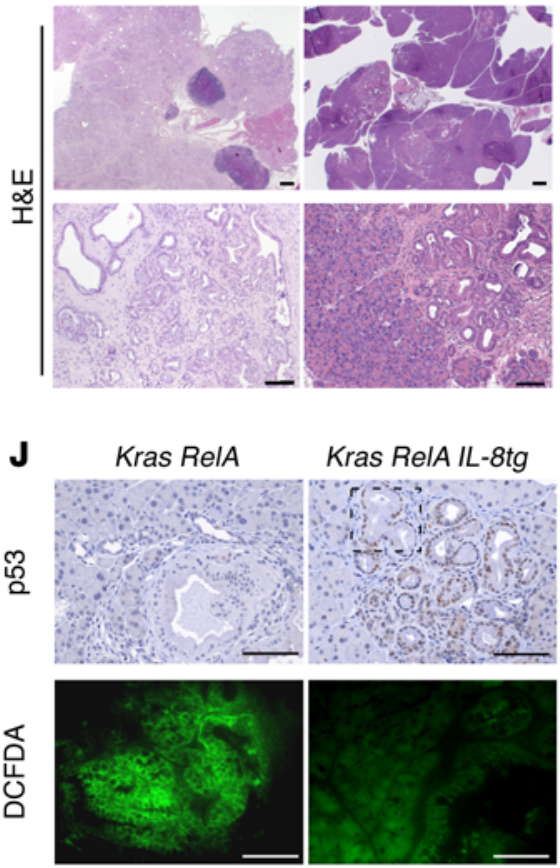

18 weeks

Kras RelA

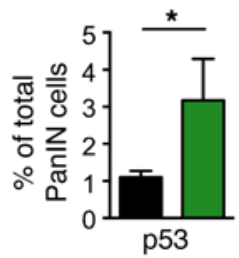

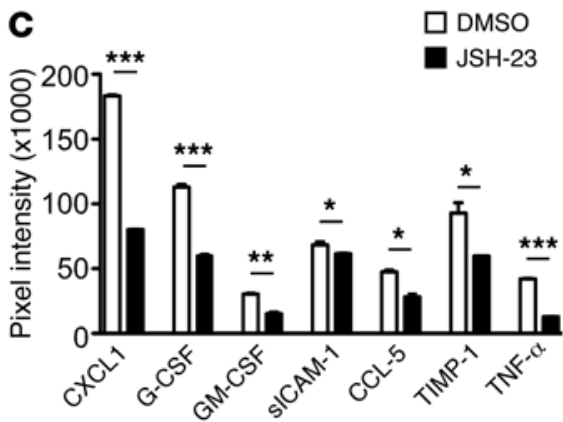

$\mathbf{F}$
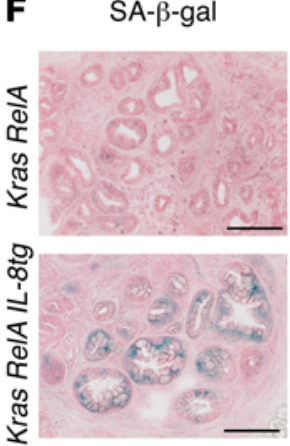

G

- Kras RelA - Kras RelA IL-8tg

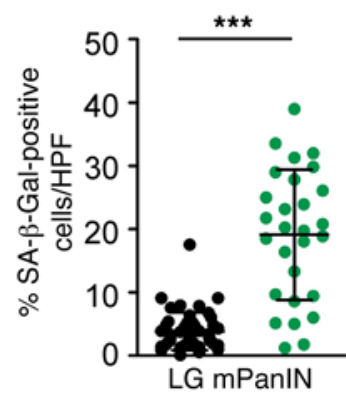

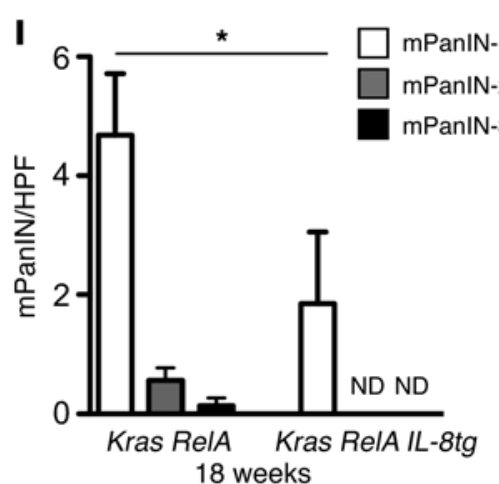

Kras RelA IL-8tg

Figure 5. Regulation of OIS by RelA is mediated by CXCL1. (A) Kras PDECs treated with vehicle control (DMSO) or an NF- $\kappa B$ inhibitor (JSH-23) were plated for the senescence assay. Scale bars: $100 \mu \mathrm{m}$. Representative images are shown. (B) Conditioned media were collected from PDECs treated with DMSO or JSH-23 for cytokine array analysis. The boxed regions on the representative blot indicate the differentially secreted cytokines. (C) Quantitative analysis of $\mathbf{B}$, representing the average of the pixel intensity of 2 independent experiments. Mean $\pm \mathrm{SD} ;{ }^{*} P<0.05,{ }^{* *} P<0.005,{ }^{* * *} P<0.0005$ by unpaired $t$ test. (D) Quantification of SA- $\beta$-Gal staining in Kras PDECs treated with DMSO or $100 \mathrm{ng} / \mathrm{ml} \mathrm{CXCL1.} \mathrm{Ratio} \mathrm{of} \mathrm{SA-} \beta$-Gal-positive Kras PDEC cultures to the total number of Kras PDECs was counted per $\times 100$ optical high-power field (HPF). Mean \pm SD; ${ }^{* *} P<0.0005$ by unpaired $t$ test. (E) Representative photomicrographs showing in situ Cxcl1 mRNA in ductal lesions (black arrowhead), in acinar cells (white arrowhead), and in immune cells (black arrow). Scale bars: 50 $\mu \mathrm{m}$. (F) Representative SA- $\beta$-Gal staining. Scale bars: $100 \mu \mathrm{m}$. (G) Ratio of SA- $\beta$-Gal-positive cells to the total number of mPanIN cells in 18 -week-old Kras RelA ( 5 mice) and Kras RelA IL-8tg (4 mice) was counted per $\times 200$ optical field (HPF). Mean \pm SD; $n \geq 28 ;{ }^{* *} P<0.0001$ by Mann-Whitney test; $n$, number of HPF. (H) Representative H\&E staining. Scale bars: $100 \mu \mathrm{m}$. (I) Numbers of mPanINs were counted per $\times 200$ optical HPF. Mean \pm SD; $n \geq 3$; ${ }^{*} P<0.05$ by unpaired $t$ test; N.D., not detectable; $n$, number of mice. (J) Representative immunohistological analysis for $p 53$ and $2^{\prime}, 7^{\prime}$-dichlorofluorescein diacetate (DCFDA) from Kras RelA $(n=3)$ and Kras RelA IL-8tg $(n=3)$ mice. Scale bars: $50 \mu \mathrm{m}$. Quantification of p53 positivity shown at the right. Mean \pm SD; ${ }^{*} P<0.05$ by unpaired $t$ test; $n$, number of mice. 
A

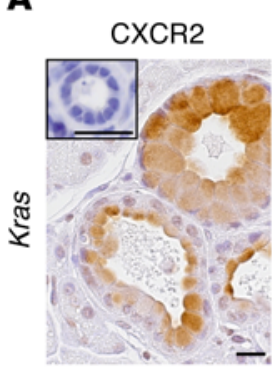

B

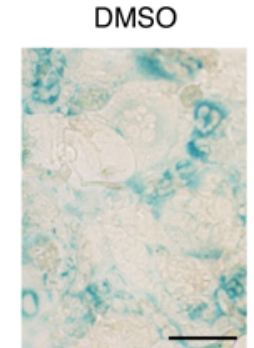

CXCL1

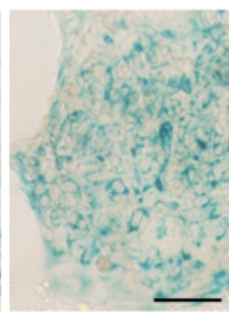

$\mathrm{CXCL} 1+$ SB225002

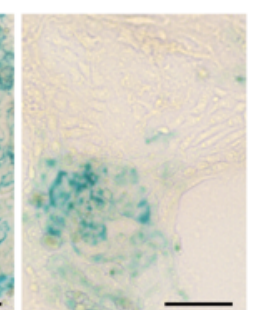

E

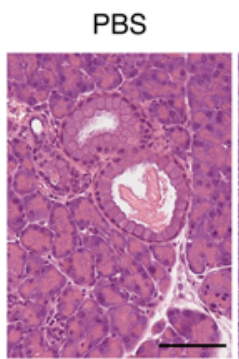

LG mPanIN

C

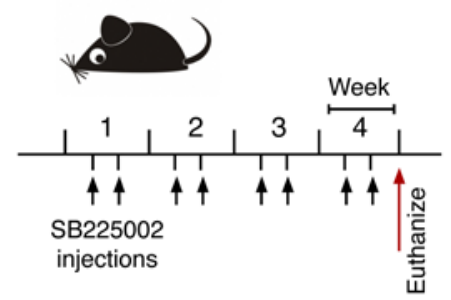

SB225002

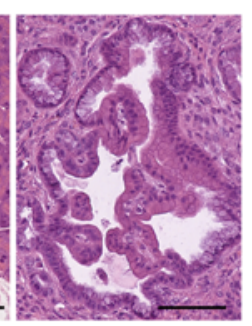

HG mPanIN

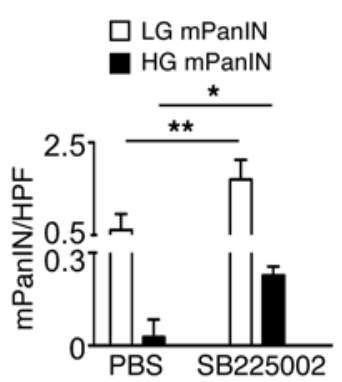

F

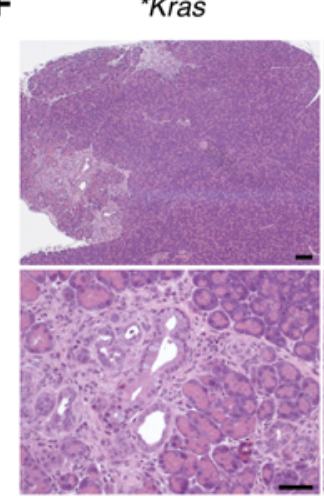

LG mPanIN

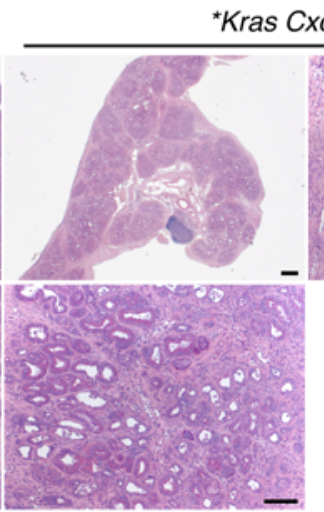

LG mPanIN

G
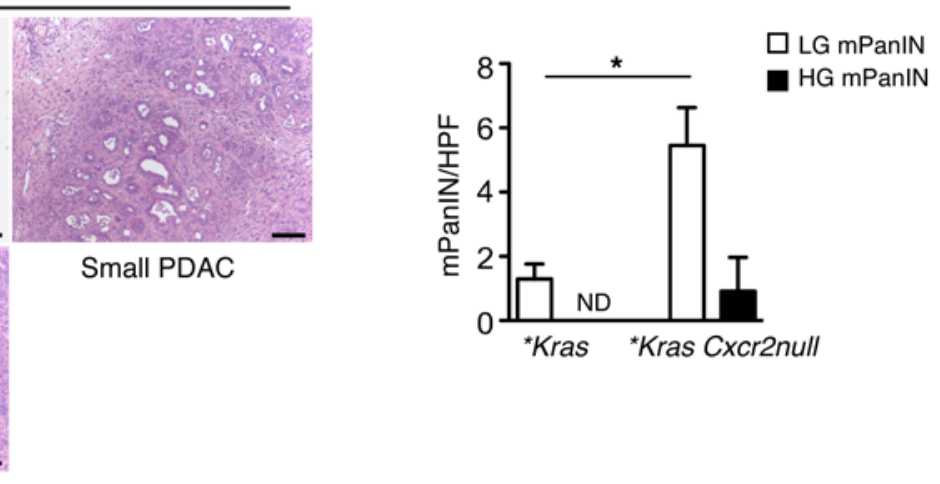

Figure 6. Reinforcement of OIS depends on CXCL1/CXCR2 axis. (A) Representative immunohistological staining for CXCR2 in mPanIN lesions from Kras pancreatic tissue and in normal ductal epithelium (inset). Scale bar: $20 \mu \mathrm{m}$. (B) Kras PDECs treated with vehicle control (DMSO), CXCL1, or a combination of CXCL1 and a CXCR2 inhibitor (SB225002) were plated for the senescence assay. Scale bar: $100 \mu \mathrm{m}$. (C) Injection protocol: Each injection contained $0.5 \mathrm{mg}$ CXCR2 inhibitor (SB225002) per kilogram body weight. Mice were treated 2 times a week over 4 weeks. One injection was administrated at each injection day. Animals were euthanized and dissected 4 days after the last injection. (D) Detection of senescence in pancreatic cryosections from 9-week-old Kras mice treated with PBS as vehicle or CXCR2 inhibitor (SB225002), using SA- $\beta$-Gal as a marker. Scale bar: $50 \mu$ m. Ratio of SA- $\beta$-Gal-positive mPanIN cells to the total number of mPanIN cells in low-grade mPanIN (LC MPanIN) in 9-week-old Kras mice treated with PBS as vehicle or CXCR2 inhibitor (SB225002) was counted per $\times 200$ optical high-power field (HPF). Mean \pm SEM; $n \geq 4$; ${ }^{*} P<0.05$ by Mann-Whitney test; $n$, number of mice. (E) Representative H\&E staining of pancreata from 9-week-old Kras mice after treatment with SB225002 or PBS. Scale bar: $50 \mu \mathrm{m}$. Number of low-grade (LG) or high-grade (HG) mPanIN in 9-week-old Kras mice treated with SB225002 or PBS per $\times 200$ optical field. Mean \pm SD; $n \geq 4 ;{ }^{*} P<0.05,{ }^{* *} P<0.005$ by unpaired $t$ test; $n$, number of mice. (F) Representative H\&E staining of pancreata from 18-week-old *Kras and ${ }^{*}$ Kras CXCR2null mice. Scale bars: $500 \mu \mathrm{m}, 100 \mu \mathrm{m}$. (G) Numbers of low-grade (LG) and high-grade (HG) mPanIN in 18-week-old ${ }^{*} K$ ras $(n=2)$ and ${ }^{*} K r a s ~ C x c r 2 n u l l ~(n=3)$ mice were counted per $\times 200$ optical field (HPF). Mean $\pm \mathrm{SD} ;{ }^{*} P<0.05$ by unpaired $t$ test; N.D., not detectable; $n$, number of mice.

A total of 390 gene sets were shown to be upregulated, 35 of which were significantly induced in Kras RelA compared with Kras pancreata (Supplemental Figure 3B; gene set enrichment analysis). Furthermore, NF-KB-mediated transcriptional pathways were highly enriched in Kras but not in Kras RelA pancreata (12\% in Kras vs. none in Kras RelA) (Figure 3A). Similarly, immunofluorescence staining revealed nuclear RelA in early mPanIN lesions and a few adjacent acinar cells of 4-week-old Kras mice, while Kras RelA pancreata were negative for nuclear RelA (Figure 3B). Moreover, "chemokine signaling pathways" (normalized enrichment score[NES] $1.5 ; P=0.002$ ) and "cyto- kine-cytokine receptor interaction pathways" (NES $=1.74 ; P<$ 0.001 ) were highly enriched in Kras but not in Kras RelA pancreata (Figure 3A). The largest group of upregulated transcription factor signatures in Kras mice was associated with proliferation, while signatures belonging to cell differentiation were highest in the Kras RelA group (Figure 3A). Positive gene set enrichment analysis (GSEA) signatures of HNF1_C $(\mathrm{NES}=1.39 ; P=0.019)$ and SOX9_B1 (NES $=1.48 ; P=0.003$ ) in Kras RelA mice indicate the possibility for increased ADM formation (Figure 3A). Furthermore, cell stress-associated transcription factor signatures were significantly increased upon loss of functional RelA 
A

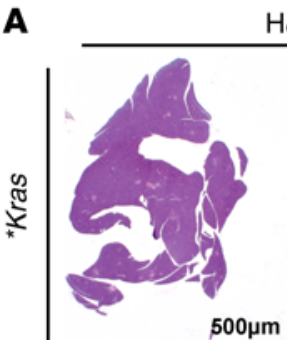

$H \& E$
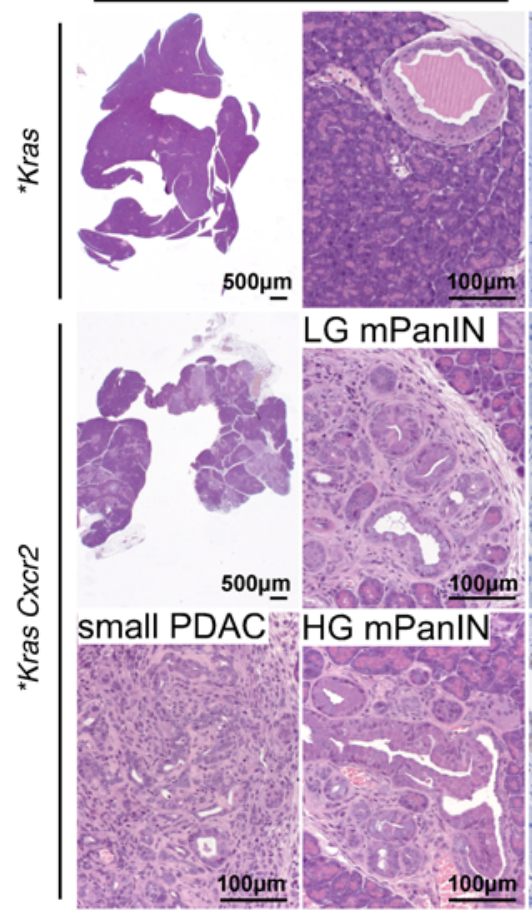

Ki-67

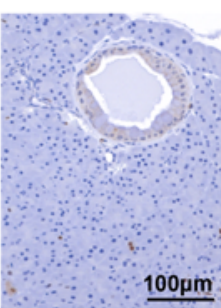

$100 \mathrm{pm}$

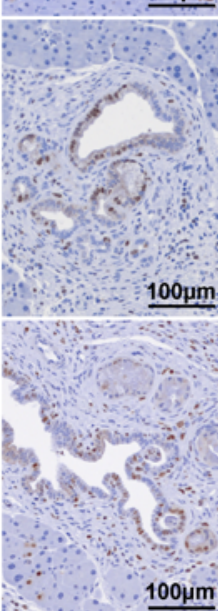

SA- $\beta-$ Gal

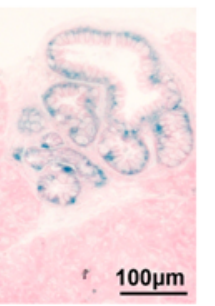

$00 \mathrm{~m}$

C

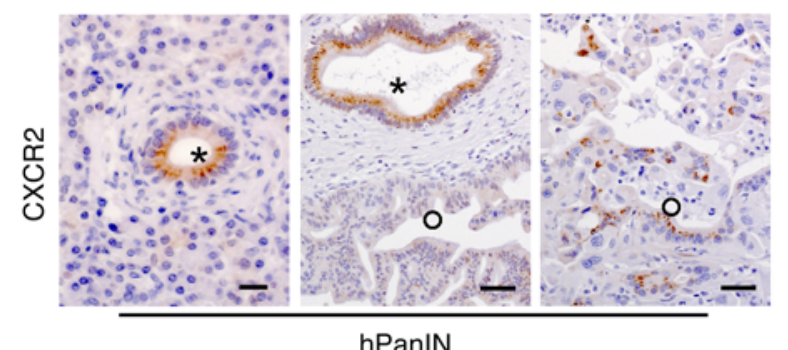

D
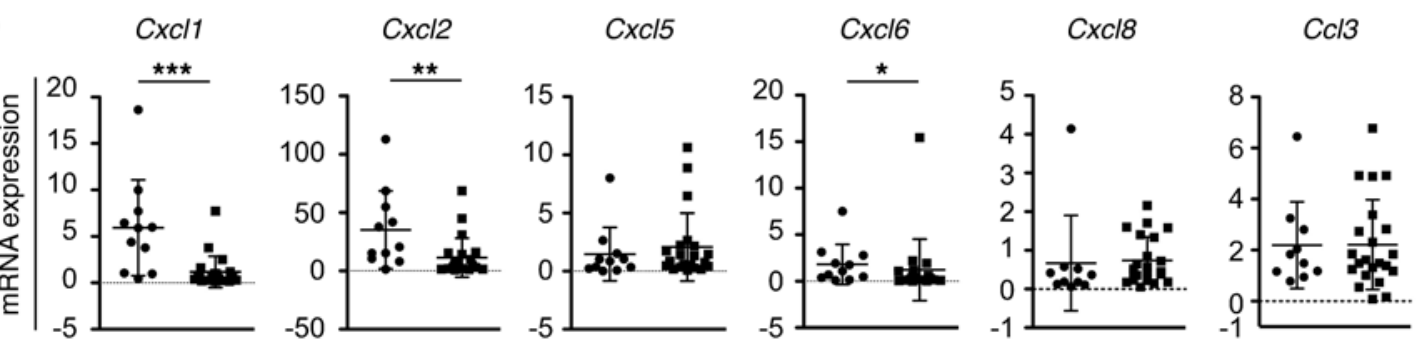

Figure 7. Pancreas-specific inactivation of CXCR2 is sufficient to bypass OIS. (A) H\&E staining, expression of Ki-67, and SA- $\beta$-Gal activity in pancreata from 18-week-old *Kras and *Kras Cxcr2 mice. Scale bars: $500 \mu \mathrm{m}, 100 \mu \mathrm{m}$. Representative images are shown. (B) Kaplan-Meier curves documenting a median survival of 195 days in ${ }^{*}$ Kras Cxcr2 mice ( $n=7$; green line) and of 162 days in ${ }^{*} K r a s ~ C X C R 2$ null mice $\left(n=40\right.$; blue line) versus 291 days in ${ }^{*} K r a s$ mice ( $n=18$; magenta line); $P=0.0488$ and $P=0.0011$ by Mantel-Cox log rank test. $n$, number of mice. (C) IHC analysis of human pancreatic cancer and tumor-adjacent tissue illustrates positive staining for CXCR2 in low-grade hPanIN (asterisk) and weak staining in high-grade hPanIN (circle) lesions. Scale bars: $20 \mu \mathrm{m}$. Representative images are shown. (D) Real-time PCR expression analysis of mRNA from microdissected human (h) low-grade (LG) PanIN cells and tumor ductal cells (hPDAC) for Cxcl1, Cxcl2, CxcI5, Cxcl6, Cxcl8, and Cc/3. Mean \pm SD; $n \geq 11 ;{ }^{*} P<0.05,{ }^{*} P<0.005,{ }^{* * *} P<0.0005$ by Mann-Whitney test; $n$, number of samples.

(Figure 3A). Indeed, IHC confirmed pronounced expression of SOX9, HNF1 $\beta$, and $\gamma$-H2AX and accumulation of ROS, which are indicative of cell differentiation and cell stress, respectively, in Kras RelA mice (Figure 3B and Supplemental Figure 3C). These effects seem to be RelA-dependent, as deletion of the superordinated kinase IKK2 in Kras mice led to significant upregulation of numerous differential gene signatures involved in myc signaling, metabolism, biosynthesis, cell stress, and cell cycle using Kyoto
Encyclopedia of Genes and Genomes (KEGG) and transcription factor signature analysis (Supplemental Table 1, A and B) (1).

Cell stress of acinar cells, for example due to inflammation, is known to drive ADM. To analyze RelA involvement in $\mathrm{ADM}$, we used an in vitro model for exocrine metaplastic conversion. Acinar pancreatic epithelial explants cultured in a collagen matrix differentiate to duct-like structures after recombinant human TGF $\alpha$ (TGF $\alpha$ ) stimulation, simulating in vivo ADM 
A

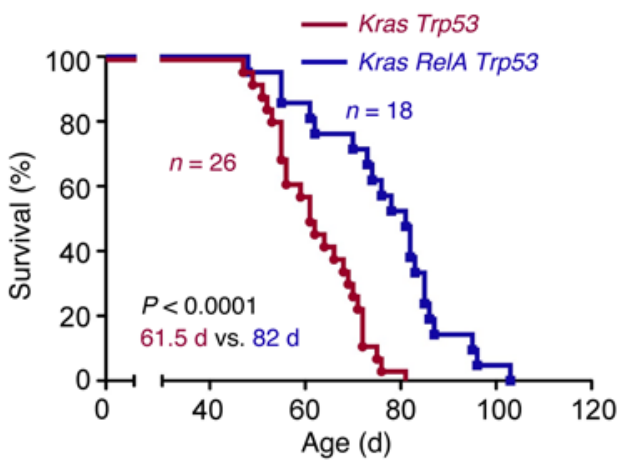

B

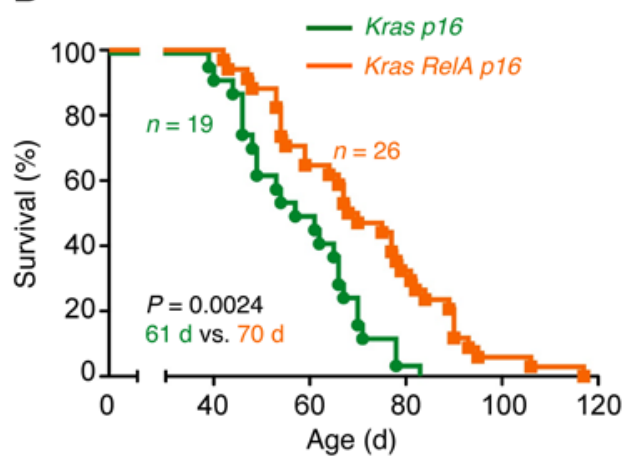

C

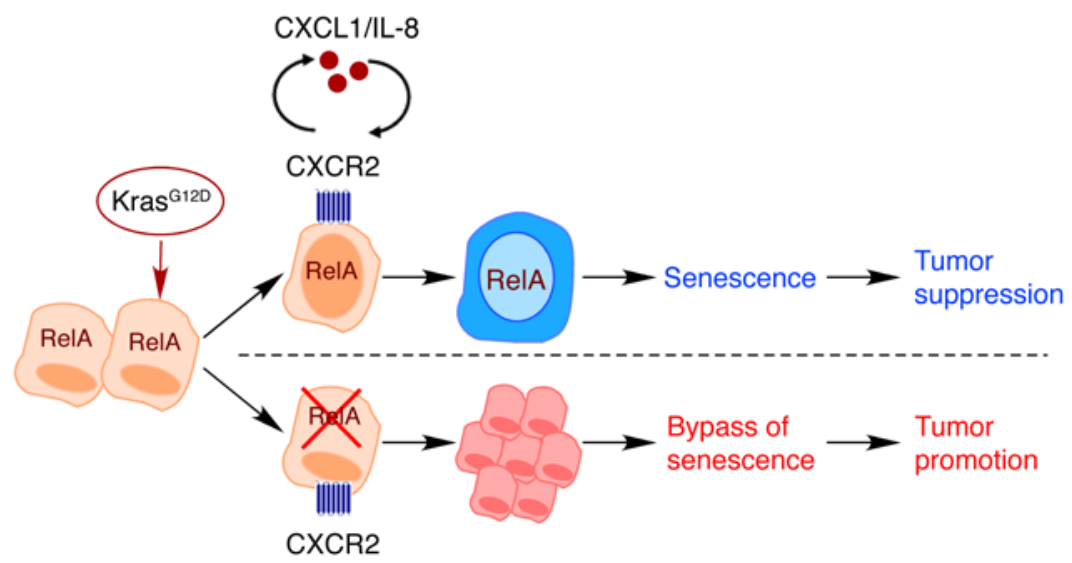

BrdU

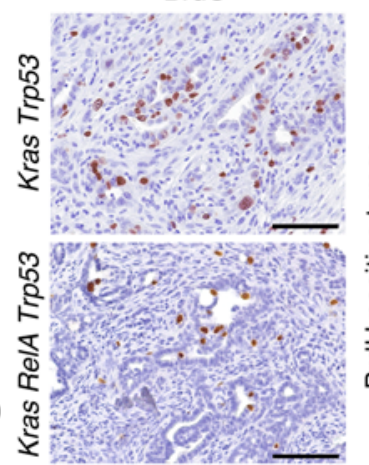

BrdU

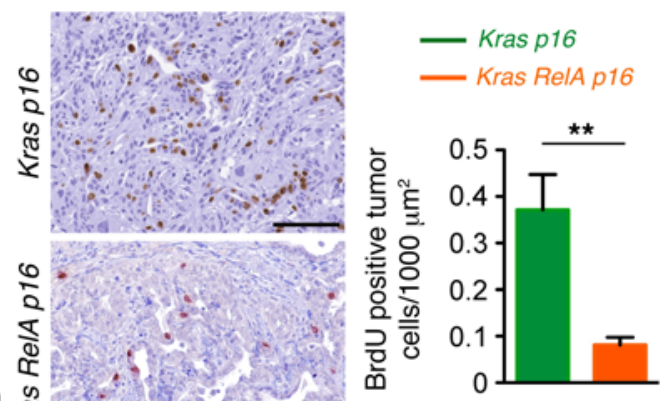

Figure 8. Abrogation of OIS in vivo illustrates dual role of RelA. (A) Kaplan-Meier curves documenting a median survival of 82 days in Kras RelA Trp53 mice ( $n=18$; blue line) versus 61.5 days in Kras Trp53 mice ( $n=26$; red line). $P<0.0001$ by Mantel-Cox log rank test; $n$, number of mice. BrdU staining of PDAC tissue samples from Kras Trp53 and Kras RelA Trp53 mice. Scale bar: $100 \mu \mathrm{m}$. The number of BrdU-positive cells was counted per tumor area. Mean \pm $\mathrm{SD} ; n=4 ;{ }^{*} P=0.0266$ by unpaired $t$ test; $n$, number of mice. (B) KaplanMeier analysis shows a median survival of 70 days in Kras RelA p16 mice ( $n=26$; orange line) versus 61 days in Kras p16 mice ( $n=19$; green line); $P=0.0024$ by Mantel-Cox log rank test; $n$, number of mice. BrdU staining of PDAC tissue samples from Kras p16 and Kras RelA p16 mice. Scale bar: $100 \mu \mathrm{m}$. The number of BrdU-positive cells was counted per tumor area. Mean $\pm S D ; n=4$; ${ }^{* *} P=0.0012$ by unpaired $t$ test; $n$, number of mice. (C) Schematic illustrating the dual role of RelA in pancreatic carcinogenesis. In early stages of tumorigenesis, the tumorsuppressive function of NF- $\mathrm{KB}$ is beneficial because it controls OIS by regulating the CXCL1/CXCR2 axis. However, as soon as OIS is bypassed during late stages of tumorigenesis, NF- $\kappa B$ supports tumor progression.
(Supplemental Figure 4A). Freshly harvested explants from pancreata of Relal/fl (hereafter referred to as RelA $A^{w t}$ ) and RelA $A^{f / f l}$ Ptfla-Cre ${ }^{e x I}$ (hereafter referred to as RelA $A^{4}$ ) littermates consisted of acinar cell clusters expressing $\alpha$-amylase, but not the duct cell marker CK8/18 (Supplemental Figure 4B). Treated with $50 \mathrm{ng} /$ $\mathrm{ml} \mathrm{TGF} \alpha$ for 5 days, $40.9 \% \pm 3.7 \%$ of the RelA ${ }^{4}$ acinar explants displayed a duct-like morphology characterized by cystic structures lined with epithelial cells expressing the duct cell marker CK19. In contrast, only $24.2 \% \pm 7.2 \%$ of acinar explants from RelA ${ }^{w t}$ pancreata transdifferentiated to duct-like structures upon TGF $\alpha$ treatment (Supplemental Figure 4, A, C, and D). To analyze ductal conversion in RelA ${ }^{w t}$ versus RelA $A^{4}$ acinar cells in an oncogenic Kras background, we isolated acinar cells from Kras and Kras RelA pancreata at an early time point to obtain maximal amount of exocrine tissue. These acinar cells were planted on collagen gel and were treated with $50 \mathrm{ng} / \mathrm{ml} \mathrm{TGF \alpha}$ for 5 days. A duct-like morphology was observed in $59.02 \% \pm 0.14 \%$ of the
Kras RelA acinar explants. In contrast, only $30.26 \% \pm 0.09 \%$ of acinar explants derived from Kras pancreata transdifferentiated to duct-like structures (Supplemental Figure 4E).

These results are consistent with a central role of RelA in controlling NF- $\kappa \mathrm{B}-$ mediated gene transcription. In the context of oncogenic Kras, RelA regulates cell stress and ADM-related pathways.

OIS in early mPanIN lesions in vivo depends on intact RelA function. Thus far, our data show that inactivation of RelA increases cell stress and facilitates mPanIN formation. However, how RelA mediates progression of mPanIN lesions to pancreatic cancer remained to be explored. Activation of the Kras oncogene can mobilize tumor suppressor networks to avert the hazard of malignant transformation. OIS represents such a network, blocking cell proliferation almost irreversibly, serving as a vital mechanism that protects against cancer. In pancreatic cancer, OIS is induced in early mPanIN, thereby impeding progression of preneoplastic lesions to PDAC (14). To identify senescent cells in situ, we 
analyzed a panel of senescence-associated markers in pancreata from Kras and Kras RelA mice. The most commonly used biomarker of OIS in vivo is an elevated activity of senescence-associated $\beta$-galactosidase (SA- $\beta-\mathrm{Gal}$ ), derived from the acidic lysosomal $\beta$-galactosidase (12). Quantification of SA- $\beta$-Gal-positive cells in low-grade mPanIN lesions from Kras and Kras RelA mice demonstrated loss of SA- $\beta$-Gal activity in Kras RelA mPanIN (Figure 4, A and B). In addition, the senescence marker decoy receptor 2 (DCR2; also known as TNFRSF10D) was significantly lower in Kras RelA pancreata than in Kras pancreata on both an mRNA expression and a protein level (Figure 4, C-E, and Supplemental Figure 5). Along the same lines, $p 53$ and $p 21$ mRNA and associated protein levels were downregulated in isolated pancreata of Kras RelA mice compared with Kras mice (Figure 4, C-E, and Supplemental Figure 5); mRNA levels of basic helix-loop-helix transcription factor $B H L H E 4 O$ (also known as dec1) also tend to be lower in Kras RelA mice (Figure 4C). Moreover, Western blot and immunohistological examinations revealed the downregulation of p16 and p19 in Kras RelA pancreata (Figure 4, D and E, and Supplemental Figure 5).

Recent studies have established that senescent cells secrete inflammatory cytokines and chemokines, extracellular proteases, and growth factors, collectively subsumed as the senescenceassociated secretory phenotype (SASP). These factors are not only known to be NF- $\mathrm{KB}$ targets, but are also required to reinforce OIS. GSEA demonstrated a loss of the SASP signature in Kras RelA mice (Figure 4F).

Further, SASP is known to contribute to the surveillance and elimination of senescent cells through a tumor-suppressive immune response during the early stages of tumorigenesis (20, 21). To characterize the immune response in Kras and Kras RelA mice during pancreatic carcinogenesis, we digested the pancreata of 8-week-old mice and performed FACS analyses. In Kras RelA pancreata total macrophage population was significantly increased as shown by FACS using general macrophage markers $\left(\mathrm{CD} 11 \mathrm{~b}^{+} \mathrm{F} 4 / 80^{+}\right)$(Supplemental Figure 6, A and B) and IHC (Supplemental Figure 6C). Among the total macrophage population, myeloid-derived suppressor cells (MDSCs; CD11 $\mathrm{b}^{+} \mathrm{Gr}-1^{+}$), typically abundant within the pancreatic cancer microenvironment and implicated in immunosuppression, were more prominently found in neoplastic pancreata of Kras RelA than in Kras mice (Supplemental Figure 6D). FACS analysis further demonstrated an enrichment of macrophages expressing the additional M2 marker CD206 (CD206 $\left.{ }^{+} \mathrm{CD} 11 b^{+} \mathrm{F} 4 / 80^{+}\right)$in Kras RelA pancreata, indicative of an M2 polarization (Supplemental Figure 6, E and F). Furthermore, deletion of Rela in the pancreas of Kras mice led to decreased expression of genes associated with M1 activation, namely Ifng, Tnf, Il12b, Cox2, and Il23a (Supplemental Figure 6G).

Thus, our data indicate that RelA activation is required to reinforce OIS in mPanIN cells, which also impacts immune response. Deletion of Rela in neoplastic Kras pancreata supports a protumorigenic microenvironment. Subsets of protumorigenic M2 macrophages and MDSCs were found to be increased in the stroma of Kras RelA mice.

Activation of RelA-dependent signaling promotes OIS through CXCL1. Since OIS is induced in early mPanIN lesions, we isolated primary pancreatic duct epithelial cells (PDEC Kras) from Kras pancreata. Treatment of these cells with a specific NF- $\kappa \mathrm{B}$ inhibitor (JSH-23) was accompanied by decreased SA- $\beta$-Gal activity (Figure $5 \mathrm{~A}$ and Supplemental Figure 7A). JSH-23 has been demonstrated to block nuclear translocation and transcription activity of RelA (22). Given the documented effect of NF- $\kappa B$ on the expression of immune mediators (11), we used antibody arrays to monitor the expression profile of secreted cytokines and chemokines in conditioned media from PDEC Kras and PDEC Kras treated with JSH-23. As expected, inhibition of nuclear translocation and transcriptional activity of RelA specifically decreased the expression of certain inflammatory mediators, including G-CSF, GM-CSF, sICAM-1, CCl-5, TIMP-1, and TNF- $\alpha$ (Figure 5, B and C). Of the 40 cytokines and chemokines represented in this panel, CXCL1 (also known as $\mathrm{KC}$ ) was most robustly downregulated in PDEC Kras treated with JSH-23 (Figure 5, B and C). The decrease in CXCL1 protein levels was corroborated by an increased SA- $\beta$-Gal activity in PDEC Kras incubated with CXCL1 for 48 hours (Figure 5D). Next, we investigated the involvement of CXCL1 in vivo by determining the cellular localization of Cxcl1 mRNA expression in pancreata from 13-week-old Kras and Kras RelA mice using the RNAscope method for in situ mRNA detection. In Kras pancreata, Cxcl1 mRNA expression was moderately to strongly present in the cytoplasm of most duct cells in mPanIN lesions, in acinar cells around mPanIN lesions, and in immune cells. Interestingly, Cxcl1 mRNA was present in immune cells, only faintly present in a few duct cells, and absent in the majority of acinar cells in Kras RelA pancreata (Figure $5 \mathrm{E})$. To determine the relevance of the observed link between the activation of RelA and CXCL1-dependent OIS, we used transgenic mice expressing $I L 8$, the human orthologue of murine CXCL1 [ $T g(I L 8)$, hereafter referred to as $I L-8 t g]$. $I L-8 t g$ mice carry a bacterial artificial chromosome that encompasses the entire human IL8 gene, including its regulatory elements (23). This mouse line was crossed to the Kras RelA strain to obtain LSL-Kras ${ }^{G 12 D}$ Rela ${ }^{f / f l} I L-8 T g$ Ptfla-Cre ${ }^{e x l}$ mice (hereafter referred to as Kras RelA $I L-8 \operatorname{tg}$ mice). Analysis and quantification of SA- $\beta$-Gal activity demonstrated a significantly higher percentage of SA- $\beta$-Gal-positive cells in low-grade lesions arising from Kras RelA IL-8tg pancreata compared with Kras RelA animals (Figure 5, F and G). Notably, IL-8 per se was sufficient to increase the number of SA- $\beta$-Gal-positive cells in low-grade mPanIN and to impede mPanIN progression in Kras animals (Supplemental Figure 7, B-D). But most strikingly, the phenotype of accelerated progression of mPanIN lesions in Kras RelA mice was nearly totally abrogated in the context of IL-8 expression (Figure 5, H and I). Coinciding with reinforcement of OIS and blocked mPanIN progression, induction of p53 in mPanIN was strongly detectable (Figure 5J). In line with the antioxidative function of $\mathrm{p} 53$, accumulation of ROS in mPanIN lacking RelA was also diminished in Kras RelA IL-8tg mice (Figure 5J).

Thus, these results strongly support the notion that CXCL1 contributes to the regulation of OIS via RelA activation in vivo and in vitro.

Systemic pharmacological and genetic inactivation of CXCR2 abrogates OIS and accelerates pancreatic carcinogenesis. Since CXCL1 acts through the chemokine receptor CXCR2, which is strictly expressed in PanIN lesions, but not in normal ductal epithelium, we then investigated whether CXCR2 is instrumental for reinforcement of OIS (Figure 6A). To this end, we treated 
PDEC Kras with CXCL1 or with a combination of CXCL1 and a CXCR2 antagonist (SB225002) in vitro. The results demonstrated increased SA- $\beta$-Gal activity in PDEC Kras treated with CXCL1, but decreased SA- $\beta-$ Gal activity in PDEC Kras treated with a combination CXCL1 and SB225002 (Figure 6B). To examine the relevance of the CXCR2 receptor in vivo, we treated 5-week-old Kras mice with SB225002 according to the protocol shown in Figure 6C or crossed LSL-Kras ${ }^{G 12 D} \mathrm{Pdx1}$-Cre mice (referred to as ${ }^{*} \mathrm{Kras}$ mice) to the $\mathrm{CxCr}^{-/-}$mouse line (LSL-Kras ${ }^{\mathrm{Gl2D}} \mathrm{Pdx1}$-Cre Cxcr2 ${ }^{-/-}$mice, referred to hereafter as ${ }^{\star} \mathrm{Kras} C \mathrm{CCr} 2 \mathrm{null}$ ). The $\mathrm{Cxcr} 2^{-/-}$mouse line lacks the receptor in the whole organism. We observed a dramatically decreased SA- $\beta$-Gal activity in low-grade mPanIN of the SB225002-treated group (Figure 6D) alongside accelerated tumorigenesis (Figure 6E). Similarly, ${ }^{\star}$ Kras Cxcr 2 null mice revealed accelerated mPanIN progression; even some focal cancers developed in 18-week-old mice, which is normally not seen in ${ }^{\star}$ Kras mice at this age (Figure 6, F and G).

Thus, our data suggest that either pharmacological or genetic systemic inactivation of CXCR2 leads to prevention of OIS, which in turn leads to accelerated pancreatic carcinogenesis.

Pancreas-specific inactivation of CXCR2 is sufficient to prevent OIS. To further corroborate the relevance of mPanIN-restricted CXCR2 for OIS in preneoplastic lesions we generated an additional ${ }^{*}$ Kras mouse line, lacking CXCR2 in the pancreas. To this end, ${ }^{*}$ Kras mice were crossed to floxed $\mathrm{Cxcr} 2$ mice to obtain the LSL$\mathrm{Kras}^{\mathrm{G12D}} \mathrm{Cxcr} 2^{f l / f l} \mathrm{Pdx1}$-Cre line (referred to as ${ }^{\star} \mathrm{Kras}$ Cxcr2 mice). Loss of CXCR2 in the pancreas resulted in impaired OIS and subsequently in increased proliferation (Figure 7A and Supplemental Figure 8, A and B). Histologically, mPanIN progression in ${ }^{\star}$ Kras Cxcr2 mice was accelerated (Supplemental Figure 8C). Notably, some ${ }^{*}$ Kras $\mathrm{Cxcr} 2$ mice developed focal cancers similar to those in ${ }^{\star}$ Kras mice lacking systemic CXCR2 expression. These observations were paralleled by decreased survival of ${ }^{*} \mathrm{Kras}$ mice lacking CXCR2 either in mPanIN lesions (green line) or in the whole organism (blue line) (Figure 7B). To determine the relevance of the CXCL1/CXCR2 axis for the human disease, we stained human pancreatic cancer specimens for CXCR2 (Figure 7C). Similarly to our observations in mice, expression of CXCR2 was detectable in low-grade, but lost in high-grade, human PanIN (hPanIN) lesions. Transcript analysis of microdissected low-grade hPanIN lesions and human pancreatic cancer cells revealed significantly increased expression levels of Cxcl1, Cxcl2, and Cxcl6 in low-grade hPanIN compared with human PDAC specimens (Figure 7D).

Moreover, we also investigated the expression of CXCR2 together with Ki-67, p53, and p16 in human normal pancreatic ducts as well as low-grade (hPanIN-1/2) and high-grade (hPanIN-3) hPanIN lesions by IHC. Supplemental Figure 8D demonstrates that CXCR2 and p16 decline as hPanIN lesions progress. In accordance with the induction of senescence in low-grade lesions, proliferation of these ducts is reduced (Supplemental Figure 8D). Conversely, p53 overexpression, which serves as a marker for the presence of p53 mutations, occurs only in high-grade hPanIN-3 lesions but is absent in low-grade hPanIN lesions (Supplemental Figure 8D), suggesting that expression of CXCR2 shares similarities with markers of OIS in human premalignant lesions (24).

These experiments demonstrate a direct impact of CXCR2 in ductal cells in the reinforcement of OIS in preneoplastic lesions during pancreatic carcinogenesis in a mouse model. Our data further provide evidence for the importance of the CXCL1/CXCR2 axis for pancreatic tumorigenesis in humans alike.

Reinforcement of OIS determines the dual role of RelA during pancreatic carcinogenesis. So far, our data indicate the significance of RelA in CXCL1/CXCR2-dependent OIS and its role as a tumor suppressor during the early stages of pancreatic tumorigenesis. To further genetically corroborate the implication of RelA in this novel and early mechanism of tumor surveillance, we took advantage of murine PDAC models that progress to pancreatic cancer via bypassing OIS. Since functional studies have demonstrated that senescence requires the $\mathrm{p} 53$ and $\mathrm{p} 16 / \mathrm{Rb}$ pathways $(25,26)$, and their abrogation is necessary to bypass senescence, we crossed floxed RelA mice to Kras mice that lacked p53 or p16 in the pancreas (16). All mice progressed to PDACs. As expected, in the absence of OIS (data not shown), survival analysis demonstrated that survival of LSL-Kras ${ }^{G 12 D}$ Rela $^{f / f l}$ Trp5 $3^{f / f l}$ Ptfla-Cre ${ }^{e x 1}$ (termed Kras RelA Trp53) and LSL-Kras ${ }^{G 12 D}$ Rela $^{f l / f l}$ Ink4a $a^{f l f l}$ Ptfla-Cre ${ }^{e x 1}$ (termed Kras RelA p16) mice was significantly longer in comparison with $L S L$ Kras $^{G 12 D} \operatorname{Trp5}^{\text {fl/fl }}$ Ptf1a-Cre ${ }^{\text {ex1 }}$ (termed Kras Trp53) and LSL-Kras ${ }^{G 12 D}$ Ink $4 a^{f l / f l} P t f 1 a-C r e^{e x 1}$ (termed Krasp16), respectively (Figure 8, A and B). Established tumors in these mice revealed decreased proliferation after functional inactivation of RelA (Figure 8, A and B).

Taken together, these data clearly demonstrate a dual role of NF- $\mathrm{B} /$ RelA activation in pancreatic carcinogenesis. In early stages of tumorigenesis, the tumor-suppressive function of NF- $\mathrm{BB}$ is beneficial because it controls OIS by regulating the CXCL1/ CXCR2 axis (Figure 8C). However, as soon as OIS is bypassed during late stages of tumorigenesis, $\mathrm{NF}-\kappa \mathrm{B}$ supports tumor progression by enhancing proliferation of the transformed pancreatic cancer cells (Figure 8C).

\section{Discussion}

Our results illustrate several mechanisms that reveal the complexity of the IKK/NF- $\mathrm{KB}$ pathway during pancreatic carcinogenesis. Furthermore, they highlight several new aspects that need to be considered, especially when exploring the use of specific IKK/ $\mathrm{NF}-\kappa \mathrm{B}$ and CXCR2 inhibitors as chemotherapeutic agents.

Recent studies have demonstrated that the components of the IKK complex IKK2 and NEMO drive progression of premalignant lesions to PDAC (1-3). However, because these kinase subunits have pleiotropic effects, it has remained unclear whether tumor promotion is directly mediated by the IKK/NF- $\kappa \mathrm{B}$ pathway (10). Some studies have confirmed the tumor-enhancing function of the NF- $\kappa$ B subunit RelA in a mouse model of pulmonary adenocarcinoma (4). However, to date, no study has implicated RelA activation in spontaneous tumor formation in vivo in the pancreas. Using an in vivo model of pancreatic cancer, we investigated the function of RelA in the $\mathrm{Kras}^{G 12 D}$ mouse line. Surprisingly, in contrast to IKK2 or NEMO, we noted rapid initiation and progression of preneoplastic lesions along with PDAC development in RelA-deficient Kras $^{G 12 D}$ mice, revealing a tumor-suppressive function for RelA. In contrast to the IKK complex, RelA is directly and immediately transferring cytoplasmic signals to the nucleus through binding of DNA, thus controlling transcription. However, blocked pancreatic carcinogenesis in IKK2/NEMO-deficient $\operatorname{Kras}^{G 12 D}$ mice was not mediated directly by the NF- $\kappa \mathrm{B}$ pathway, but through interlinked 
pathways such as Notch, KLF, or IL-1/p62 (1-3). In fact, the IKK complex provides a basis for manifold cross-talk with other signaling pathways, as well as feedback circuits. In contrast to Kras RelA mice, comparative analysis of gene signatures revealed significant differences, including upregulation of numerous nonoverlapping pathways, in Pdx1-Cre LSL-Kras ${ }^{G 12 D} I K K 2^{f / f l}$ mice (Supplemental Table 1, A and B) (1). Loss of IKK2 resulted in induction of several pathways, mainly related to metabolism and biosynthesis, whereas functional inactivation of RelA only promoted cell stress and cell differentiation pathways. This further underlines substantial differences between the components of the IKK/NF- $\kappa \mathrm{B}$ pathway. Although most studies have demonstrated a pro-oncogenic function of NF- $\mathrm{BB}$, our findings are consistent with a tumor-suppressive function, as observed in other tumor models of the skin, the liver, and lymphomas $(7,9,27,28)$.

OIS is a failsafe mechanism that prevents the development of cancer in precancerous lesions of various tumors, including PDAC (29). Malignant cells are unable to undergo senescence, because of the loss of OIS effectors, such as p53 or p16 (30). The molecular mechanisms that regulate OIS during pancreatic oncogenesis are unknown. Using various mouse models of pancreatic cancer, we have provided novel findings that show that OIS requires activation of the RelA-dependent canonical NF- $\kappa$ B pathway during $\mathrm{mPanIN}$ progression. Senescence is mediated by cell-autonomous and non-cell-autonomous mechanisms that regulate OIS in a paracrine manner. Preneoplastic ductal cells that express Kras $^{G 12 D}$ are not only the chief source of SASP components; mPanIN lesions are also the recipients, undergoing OIS through CXCR2 activation. The SASP comprises cytokines, chemokines, matrix metalloproteinases, and other proteins, most of which are regulated by NF- $\kappa B(11,31-35)$. SASP components, such as IL-1 $\beta$, IL-6, and CXCL1, were less prominently induced in Kras mice lacking RelA in the pancreas. Consequently, OIS was attenuated in these mice, whereas mice expressing $\operatorname{Kras}^{G 12 D}$ since embryonic development showed increased SA- $\beta$-Gal activity and widespread OIS in most early preneoplastic lesions (12). Our findings support the notion that induction of OIS in Kras mice necessitates the activation of RelA to regulate SASP $(11,29)$. RelA has been shown to cooperate with p53 in the execution of the senescence program (36). This interplay influences macrophage polarization and the subsequent targeting of senescent cells.

RelA also showed a potent effect on macrophage regulation. Tumor-associated macrophages can be polarized toward a classical, proinflammatory phenotype M1, or toward an alternative, anti-inflammatory $\mathrm{M} 2$ response. M1 macrophages are characterized by a proinflammatory cytokine profile and are potent killers of tumor cells, while M2 macrophages show mainly tumorenhancing functions, promoting cell survival and proliferation $(37,38,39)$. Interestingly, the senescent state triggered by $\mathrm{Kras}^{G 12 D}$ caused a significant increase in the secretion of factors known to promote M1 polarization, which is characterized by an increased propensity for cell killing and phagocytosis (40). Loss of RelA was associated with suppression of secretion of these factors and promotion with M2 polarization of macrophages and accumulation of MDSCs. MDSCs have been implicated in promoting tumor growth by suppressing antitumor immunity $(41,42)$. Interestingly, in the context of inflammation-associated tumorigenesis, recruitment of MDSCs was shown to depend on CXCL1/CXCR2. Recent work identifies CXCR2 as a new target for therapies aimed at inhibiting MDSC recruitment, providing a rationale for combining immune checkpoint inhibitors with agents designed to prevent MDSC-mediated immune suppression for cancer therapy (43). However, it seems that this mechanism is not crucial for the recruitment of MDSCs in the absence of OIS during Kras-induced pancreatic carcinogenesis. Beyond the CXCR2 ligands in mice (CXCL1, CXCL2/3, CXCL5, and CXCL7), several other factors, such as S100A8/A9, have been shown to attract myeloid cells to the tumor microenvironment (44). These factors do not only induce the accumulation of MDSCs; they are also secreted by MDSCs and by tumor cells to ensure the maintenance of functionally suppressive MDSCs within a tumor environment. Thus, rather than promoting inflammation, activation of RelA regulates the fine-tuning of tumor-inhibitory and tumor-promoting effects via SASP immune responses.

Of all SASP components, the CXCR2 ligand CXCL1 was shown to be required for OIS induction in vitro, whereas others, including IL- 6 and IL-1 $\beta$, have been shown to promote malignant phenotypes (45-47). Consistent with a previous study, PDECs that expressed $\mathrm{Kras}^{G 12 D}$ failed to undergo senescence without incubation with CXCL1 (15). CXCR2 in the pancreas of Kras mice is restricted to ductal cells. Although most studies have endorsed a pro-oncogenic function of CXCL1 and the human ortholog IL-8 in human tumors (48), our pharmacological and genetic approaches suggest a tumor surveillance role in pancreatic oncogenesis through the RelA-dependent regulation of the IL-8/CXCL1/CXCR2 axis. The CXCL1/CXCR2 axis is a critical regulatory system that reinforces growth arrest through senescence in vitro. Importantly, genetic and pharmaceutical blockage of CXCR2 in vivo mimicked a RelA-deficient phenotype, with reduced senescence and increased mPanIN progression. In contrast, overexpression of the human ortholog of IL-8 inhibited progression of preneoplastic lesions through expansion of senescent mPanIN lesions (25). However, the effects of CXCR2 on pancreatic oncogenesis appear to be context- and stage-dependent, because a recent study has demonstrated that CXCR2 promotes the development of PDAC in a mouse model (49). Notably, in this study activation of $\mathrm{Kras}^{G 12 D}$ was achieved by the Pdx1-Cre mouse line. Further, in the context of chronic inflammation-induced carcinogenesis in the colon, loss of CXCR2 suppresses inflammation-associated tumorigenesis. These effects have been attributed to the inhibition of infiltration of MDSCs into the colonic mucosa. However, in the context of spontaneous Kras-driven cancer development, CXCR2 and its ligands seem to play a central role in establishing OIS. Once OIS has been overcome, CXCR2 signaling seems to switch from a tumor suppressor to a tumor promoter (50).

In summary, we have examined the in vivo function of RelA during pancreatic oncogenesis, which comprises a dual mechanism (51-54). While on the one hand RelA inhibits the development of preneoplastic lesions by reinforcing senescence through the CXCL1/CXCR2 axis, its activation in established tumors promotes the cancer cell proliferation. Examining these contextdependent activities of RelA will be important for effective clinical use of NF- $\kappa B$ inhibitors. Furthermore, these findings underscore 
that caution should be exercised when exploring the use of pharmaceuticals targeting the CXCR2 receptor as a therapeutic option for the treatment of various solid tumors.

\section{Methods}

Mice. LSL-Kras ${ }^{G 12 D}$ knock-in, Ptfla-Creexl, RelA $A^{f l f l}$ (termed RelAt), $\operatorname{Trp} 53^{f / f l}, \operatorname{Ink} 4 a$, and $\operatorname{Tg}(I L 8)$ strains were interbred to obtain the compound mutant strains Rela ${ }^{f / f l}$ Ptfla-Cre ${ }^{e x 1}$ (termed RelA), LSL-Kras ${ }^{G 12 D}$ RelA $^{f / f l}$ Ptfla-Cre ${ }^{e x l}$ (termed Kras RelA), LSL-Kras ${ }^{\text {Gl2D }} \mathrm{Tg}(\mathrm{IL} 8)$ Ptfla-Cre ${ }^{e x 1}$ (termed Kras IL-8tg), LSL-Kras ${ }^{G 12 D}$ Relafl/fl IL-8tg Ptfla-Cre ${ }^{e x 1}$ (termed Kras RelA IL-8tg), LSL-Kras ${ }^{G 12 D}$ Trp53 ${ }^{\text {fl/fl }}$ Ptfla-Cre ${ }^{e x I}$ (termed Kras Trp53), LSL-Kras ${ }^{G 12 D}$ Rela $^{\text {fl/fl }}$ Trp53 $3^{\text {flfl }}$ Ptfla-Creexl (termed Kras RelA Trp53), LSL-Kras ${ }^{G 12 D}$ Ink4a $a^{f / f l}$ Ptfla-Creexl (termed Kras p16), and LSLKras $^{G 12 D}$ RelA $^{f l / f l}$ Ink $4 a^{f l / f l}$ Ptfla-cre ${ }^{e x l}$ (termed Kras RelA p16). Mutant LSL-Kras ${ }^{G 12 D}$ Ptfla-Cre ${ }^{e x 1}$ mice (termed Kras, the positive control) were used as control animals $(19,25,55-58)$. LSL-Kras ${ }^{G 12 D} P d x 1$-Cre (termed ${ }^{*}$ Kras), LSL-Kras ${ }^{G 12 D} \mathrm{Cxcr} 2^{f / f l} \mathrm{Pdx1}$-Cre (termed ${ }^{*} \mathrm{Kras} \mathrm{Cxcr} 2$ ), and LSLKras $^{G 12 D} \mathrm{Pdx1}$-Cre Cxcr ${ }^{-1-}$ (termed ${ }^{*} \mathrm{Kras} C x \mathrm{Cr} 2 \mathrm{null}$ ) mice were obtained from Owen J. Sansom.

RNAscope method for in situ mRNA detection. In situ detection of Cxcl1 transcripts in formalin-fixed paraffin-embedded (FFPE) pancreatic samples was performed using the RNAscope method (Advanced Cell Diagnostics) according to the manufacturer's instructions. Briefly, 4- $\mu \mathrm{m}$ FFPE pancreatic sections were pretreated with heat, deparaffinized, boiled with a pretreatment solution for $15 \mathrm{~min}$ utes, and submitted to protease digestion followed by hybridization for 2 hours with a target probe for CXCL1. Thereafter, an HRP-based signal amplification system was hybridized to the target probe before color development with 3,3'-diaminobenzidine tetrahydrochloride. Positive staining was defined as the presence of brown dots.The housekeeping gene ubiquitin $C(U b c)$ served as a positive control. The $D a p B$ gene, which is derived from a bacterial gene sequence, was used as a negative control.

Fluorescence-activated cell sorting (FACS). Single-cell suspensions were prepared from fresh pancreas and spleen. Pancreata were minced, incubated with $1.2 \mathrm{mg} / \mathrm{ml}$ collagenase type VIII (catalog C2139; Sigma-Aldrich) and $0.1 \mathrm{mg} / \mathrm{ml}$ soybean trypsin inhibitor (catalog T9003; Sigma-Aldrich) in PBS for 10 minutes at $37^{\circ} \mathrm{C}$, and then passed through a Corning 70- $\mu$ m Cell Strainer (catalog 352350; Corning). Spleens were crushed, passed through a $70-\mu \mathrm{m}$ cell strainer, washed once with PBS/2\% FCS, and treated with rbc lysis buffer (catalog R7757; Sigma-Aldrich) for 90 seconds to eliminate rbcs. Cell suspensions were stained in PBS/2\% FCS with the following antibodies: CD45 (clone 30-F11), CD3e (clone 145-2C11), CD11b (clone M1/70), F4/80 (clone BM8; all from eBioscience); CD206 (clone C068C2), CD4 (clone GK1.5) (all from BioLegend); and Gr-1 (clone RB6-8C5) (Thermo Fisher Scientific). Flow cytometry analysis was performed using a Gallios flow cytometer (Beckman Coulter) after gating and exclusion of dead cells. Data were analyzed using a software package (FlowJo).

Accession number. Microarray data are available in the ArrayExpress database (www.ebi.ac.uk/arrayexpress) under accession number E-MTAB-3436.

Supplemental Methods. For all methods not listed here, please refer to the online Supplemental Methods section.

\section{Author contributions}

HA formulated hypotheses and designed experiments. ML performed the majority of experiments and analyzed data. JM, OS, DS, GJS, and TCW provided murine models. SMW contributed to flow cytometry sorting analyses. KND and $\mathrm{HE}$ analyzed microarray data. OK, MW, and TK performed several experiments. JS, IED, and BS provided patient samples and analyzed human data. ML and HA wrote the manuscript. JM, IED, MH, JMS, TCW, and RMS edited the manuscript.

\section{Acknowledgments}

We thank Chantal Geisert and Viktoria Mayr for excellent technical assistance. This work was supported by grants from Deutsche Forschungsgemeinschaft (AL 1174/5-1 and LE 3222/1-1) and Deutsche Krebshilfe (111646).

Address correspondence to: Hana Algül, II. Medizinische Klinik, Klinikum rechts der Isar, Technische Universität München, 81675 Munich, Germany. Phone: 49.89.4140.5215; E-mail: hana. alguel@mri.tum.de.
1. Ling J, et al. KrasG12D-induced IKK2/ $\beta / \mathrm{NF}-\kappa \mathrm{B}$ activation by IL- $1 \alpha$ and $\mathrm{p} 62$ feedforward loops is required for development of pancreatic ductal adenocarcinoma. Cancer Cell. 2012;21(1):105-120.

2. Maier HJ, Wagner M, Schips TG, Salem HH, Baumann B, Wirth T. Requirement of NEMO/ IKK $\gamma$ for effective expansion of KRAS-induced precancerous lesions in the pancreas. Oncogene. 2013;32(21):2690-2695.

3. Maniati E, et al. Crosstalk between the canonical $\mathrm{NF}-\kappa \mathrm{B}$ and Notch signaling pathways inhibits Ppary expression and promotes pancreatic cancer progression in mice. JClin Invest. 2011;121(12):4685-4699.

4. Bassères DS, Ebbs A, Levantini E, Baldwin AS. Requirement of the NF-kappaB subunit p65/ RelA for K-Ras-induced lung tumorigenesis. Cancer Res. 2010;70(9):3537-3546.

5 . Xia Y, et al. Reduced cell proliferation by IKK2 depletion in a mouse lung-cancer model. Nat Cell
Biol. 2012;14(3):257-265.

6. Meylan E, et al. Requirement for NF-kappaB signalling in a mouse model of lung adenocarcinoma. Nature. 2009;462(7269):104-107.

7. Maeda S, Kamata H, Luo JL, Leffert H, Karin M. IKKbeta couples hepatocyte death to cytokinedriven compensatory proliferation that promotes chemical hepatocarcinogenesis. Cell. 2005;121(7):977-990.

8. Luedde T, et al. Deletion of NEMO/IKKgamma in liver parenchymal cells causes steatohepatitis and hepatocellular carcinoma. Cancer Cell. 2007;11(2):119-132.

9. Dajee M, et al. NF-kappaB blockade and oncogenic Ras trigger invasive human epidermal neoplasia. Nature. 2003;421(6923):639-643.

10. Perkins ND. The diverse and complex roles of NF- $\mathrm{BB}$ subunits in cancer. Nat Rev Cancer. 2012;12(2):121-132.

11. Chien Y, et al. Control of the senescence-asso- ciated secretory phenotype by NF- $\kappa \mathrm{B}$ promotes senescence and enhances chemosensitivity. Genes Dev. 2011;25(20):2125-2136.

12. Caldwell ME, et al. Cellular features of senescence during the evolution of human and murine ductal pancreatic cancer. Oncogene. 2012;31(12):1599-1608.

13. Collado M, et al. Tumour biology: senescence in premalignant tumours. Nature. 2005;436(7051):642.

14. Guerra C, et al. Pancreatitis-induced inflammation contributes to pancreatic cancer by inhibiting oncogene-induced senescence. Cancer Cell. 2011;19(6):728-739.

15. Lee KE, Bar-Sagi D. Oncogenic KRas suppresses inflammation-associated senescence of pancreatic ductal cells. Cancer Cell. 2010;18(5):448-458.

16. Morton JP, et al. Mutant p53 drives metastasis and overcomes growth arrest/senescence in pancreatic cancer. Proc Natl Acad Sci US A. 
2010;107(1):246-251.

17. Collado M, Blasco MA, Serrano M. Cellular senescence in cancer and aging. Cell. 2007;130(2):223-233.

18. Moir JA, White SA, Mann J. Arrested development and the great escape - the role of cellular senescence in pancreatic cancer. Int J Biochem Cell Biol. 2014;57:142-148.

19. Algül H, et al. Pancreas-specific RelA/p65 truncation increases susceptibility of acini to inflammation-associated cell death following cerulein pancreatitis. J Clin Invest. 2007;117(6):1490-1501.

20. Kang TW, et al. Senescence surveillance of premalignant hepatocytes limits liver cancer development. Nature. 2011;479(7374):547-551.

21. Krizhanovsky V, et al. Senescence of activated stellate cells limits liver fibrosis. Cell. 2008;134(4):657-667.

22. Hu C, Sun L, Hu Y, Lu D, Wang H, Tang S. Functional characterization of the NF-kappaB binding site in the human NOD2 promoter. Cell Mol Immunol. 2010;7(4):288-295.

23. Asfaha S, et al. Mice that express human interleukin-8 have increased mobilization of immature myeloid cells, which exacerbates inflammation and accelerates colon carcinogenesis. Gastroenterology. 2013;144(1):155-166.

24. Schwitalla S, et al. Loss of p53 in enterocytes generates an inflammatory microenvironment enabling invasion and lymph node metastasis of carcinogen-induced colorectal tumors. Cancer Cell. 2013;23(1):93-106.

25. Campisi J. Aging, cellular senescence, and cancer. Annu Rev Physiol. 2013;75:685-705.

26. Tchkonia T, Zhu Y, van Deursen J, Campisi J, Kirkland JL. Cellular senescence and the senescent secretory phenotype: therapeutic opportunities. J Clin Invest. 2013;123(3):966-972.

27. Jing $\mathrm{H}$, et al. Opposing roles of NF- $\mathrm{KB}$ in anti-cancer treatment outcome unveiled by cross-species investigations. Genes Dev. 2011;25(20):2137-2146.

28. Maeda S, et al. IкB kinase $\beta /$ nuclear factor $-\kappa B$ activation controls the development of liver metastasis by way of interleukin- 6 expression. Hepatology . 2009;50(6):1851-1860.

29. Rodier F, Campisi J. Four faces of cellular senescence. JCell Biol. 2011;192(4):547-556.

30. Singh SK, Ellenrieder V. Senescence in pancreatic carcinogenesis: from signalling to chromatin remodelling and epigenetics. Gut.
2013;62(9):1364-1372.

31. Acosta JC, et al. A complex secretory program orchestrated by the inflammasome controls paracrine senescence. Nat Cell Biol. 2013;15(8):978-990.

32. Coppé JP, Desprez PY, Krtolica A, Campisi J. The senescence-associated secretory phenotype: the dark side of tumor suppression. Annu Rev Pathol. 2010;5:99-118.

33. Coppé JP, et al. Senescence-associated secretory phenotypes reveal cell-nonautonomous functions of oncogenic RAS and the p53 tumor suppressor. PLoS Biol. 2008;6(12):2853-2868.

34. Pérez-Mancera PA, Young AR, Narita M. Inside and out: the activities of senescence in cancer. Nat Rev Cancer. 2014;14(8):547-558.

35. Young AR, Narita M. SASP reflects senescence. EMBO Rep. 2009;10(3):228-230.

36. Lujambio A, et al. Non-cell-autonomous tumor suppression by p53. Cell. 2013;153(2):449-460.

37. Sica A, Bronte V. Altered macrophage differentiation and immune dysfunction in tumor development. J Clin Invest. 2007;117(5):1155-1166.

38. Murray PJ, Wynn TA. Protective and pathogenic functions of macrophage subsets. Nat Rev Immunol. 2011;11(11):723-737.

39. Mantovani A, Biswas SK, Galdiero MR, Sica A, Locati M. Macrophage plasticity and polarization in tissue repair and remodelling. J Pathol. 2013;229(2):176-185.

40. Biswas SK, Mantovani A. Macrophage plasticity and interaction with lymphocyte subsets: cancer as a paradigm. Nat Immunol. 2010;11(10):889-896.

41. Khaled YS, Ammori BJ, Elkord E. Myeloidderived suppressor cells in cancer: recent progress and prospects. Immunol Cell Biol. 2013;91(8):493-502.

42. Lindau D, Gielen P, Kroesen M, Wesseling P, Adema GJ. The immunosuppressive tumour network: myeloid-derived suppressor cells, regulatory $\mathrm{T}$ cells and natural killer T cells. Immunology. 2013;138(2):105-115.

43. Highfill SL, et al. Disruption of CXCR2-mediated MDSC tumor trafficking enhances anti-PD1 efficacy. Sci Transl Med. 2014;6(237):237ra67.

44. Gebhardt C, Nemeth J, Angel P, Hess J. S100A8 and S100A9 in inflammation and cancer. Biochem Pharmacol. 2006;72(11):1622-1631.

45. Acosta JC, et al. Chemokine signaling via the
CXCR2 receptor reinforces senescence. Cell. 2008;133(6):1006-1018

46. Lesina M, et al. Stat3/Socs3 activation by IL-6 transsignaling promotes progression of pancreatic intraepithelial neoplasia and development of pancreatic cancer. Cancer Cell. 2011;19(4):456-469.

47. Quante $\mathrm{M}$, et al. Bile acid and inflammation activate gastric cardia stem cells in a mouse model of Barrett-like metaplasia. Cancer Cell. 2012;21(1):36-51.

48. Matsuo Y, et al. CXCL8/IL-8 and CXCL12/ SDF-1 $\alpha$ co-operatively promote invasiveness and angiogenesis in pancreatic cancer. Int J Cancer. 2009;124(4):853-861.

49. Ijichi $\mathrm{H}$, et al. Inhibiting Cxcr2 disrupts tumorstromal interactions and improves survival in a mouse model of pancreatic ductal adenocarcinoma. J Clin Invest. 2011;121(10):4106-4117.

50. Katoh H, Wang D, Daikoku T, Sun H, Dey SK, Dubois RN. CXCR2-expressing myeloidderived suppressor cells are essential to promote colitis-associated tumorigenesis. Cancer Cell. 2013;24(5):631-644.

51. Burgess DJ. Senescence. NF- $\kappa$ B shows its beneficial side. Nat Rev Cancer. 2011;11(12):832-833.

52. Klein U, Ghosh S. The two faces of NF- $\kappa$ B signaling in cancer development and therapy. Cancer Cell. 2011;20(5):556-558.

53. Perkins ND. NF-kB: tumor promoter or suppressor? Trends Cell Biol. 2004;14(2):64-69.

54. Tergaonkar V, Perkins ND. p53 and NF-кB crosstalk: IKK $\alpha$ tips the balance. Mol Cell. 2007;26(2):158-159.

55. Hingorani SR, et al. Preinvasive and invasive ductal pancreatic cancer and its early detection in the mouse. Cancer Cell. 2003;4(6):437-450.

56. Marino S, Vooijs M, van Der Gulden H, Jonkers J, Berns A. Induction of medulloblastomas in p53null mutant mice by somatic inactivation of $\mathrm{Rb}$ in the external granular layer cells of the cerebellum. Genes Dev. 2000;14(8):994-1004.

57. Nakhai H, et al. Ptfla is essential for the differentiation of GABAergic and glycinergic amacrine cells and horizontal cells in the mouse retina. Development. 2007;134(6):1151-1160.

58. Aguirre AJ, et al. Activated Kras and Ink4a/ Arf deficiency cooperate to produce metastatic pancreatic ductal adenocarcinoma. Genes Dev. 2003;17(24):3112-3126 